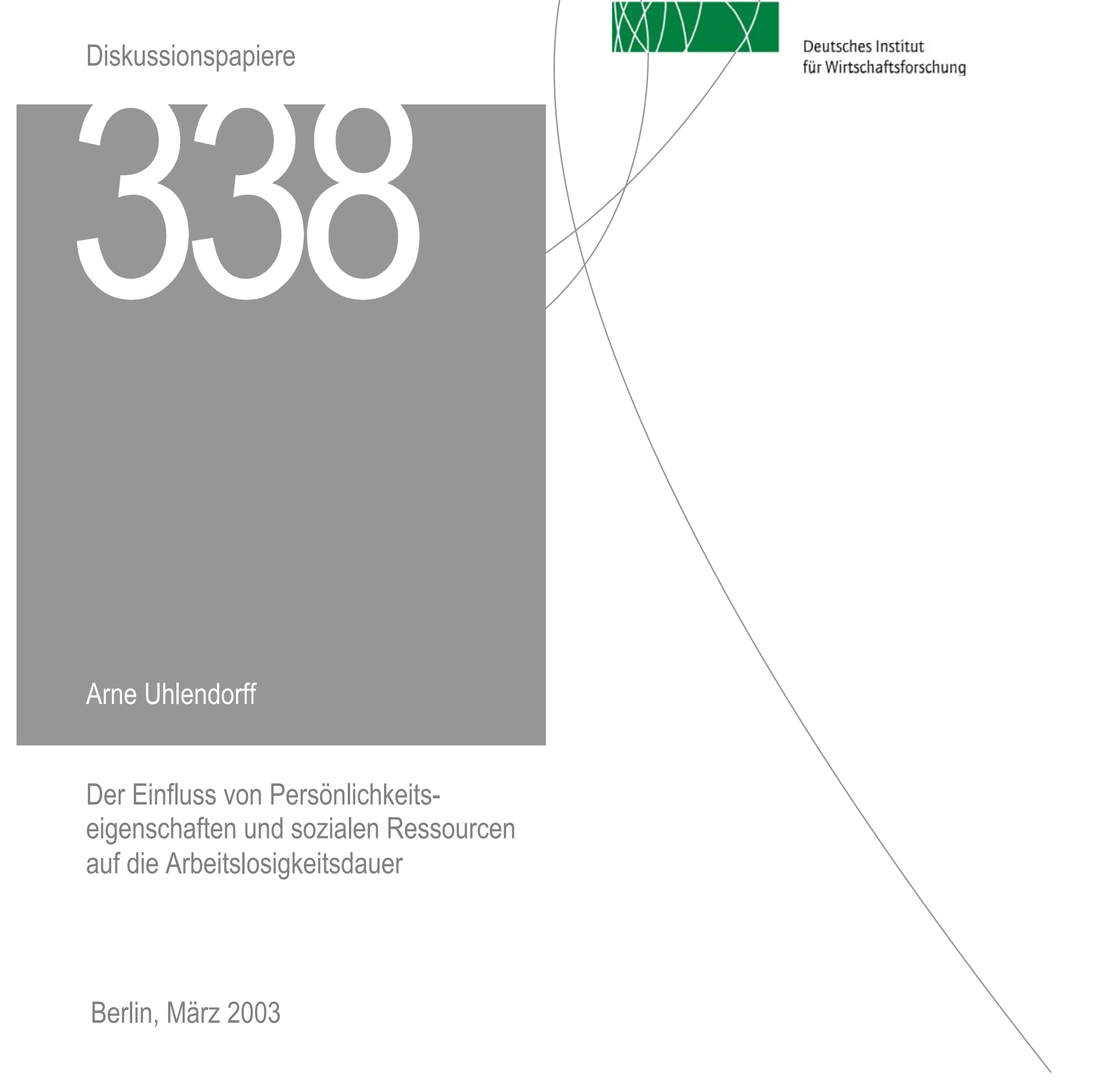


Die in diesem Papier vertretenen Auffassungen liegen ausschließlich in der Verantwortung des Verfassers und nicht in der des Instituts.

\section{DIW Berlin}

Deutsches Institut für Wirtschaftsforschung

Königin-Luise-Str. 5

14195 Berlin

Tel. $03089789-0$

Fax $03089789-200$

www.diw.de

ISSN 1619-4535 


\title{
Der Einfluss von Persönlichkeitseigenschaften und sozialen Ressourcen auf die Arbeitslosigkeitsdauer
}

\author{
Arne Uhlendorff* \\ DIW Berlin
}

Februar 2003

\begin{abstract}
Zusammenfassung
Untersucht wird der Einfluss des Humankapitals, der Arbeitsmarktlage, der familiären Situation sowie der Persönlichkeitseigenschaften und sozialen Ressourcen auf die individuelle Arbeitslosigkeitsdauer anhand des Sozioökonomischen Panels (SOEP) in West- und Ostdeutschland. Es wird eine unproportionale Cox-Regression mit Interaktionseffekten ausgewählter Prädiktoren mit der Zeit geschätzt. Die Ergebnisse vergleichbarer Analysen werden bestätigt und es zeigt sich, dass die Berücksichtigung der üblicherweise vernachlässigten Persönlichkeitseigenschaften und sozialen Ressourcen neue Erkenntnisse liefert. In Westdeutschland finden mobile und internal kontrollierte Arbeitslose schneller eine neue Beschäftigung, während in Ostdeutschland soziales Kapital positive Auswirkungen auf die Beschäftigungswahrscheinlichkeit hat.
\end{abstract}

\begin{abstract}
The influence of human capital, the local unemployment rate, the family situation, personal characteristics and the social resources on the individual length of unemployment in Germany are analysed with data from the German Socio-Economic Panel (GSOEP). Because of interactions of some covariables with time, an unproportional Cox-regression is estimated. This analysis confirms the results of previous studies, and the consideration of personal characteristics and social resources leads to new additional findings. In West-Germany mobile persons and actors with internal control find a job more easily while in East-Germany social capital has a positive influence on the probability of reemployment.
\end{abstract}

* DIW Berlin, Deutsches Institut für Wirtschaftsforschung, Königin-Luise-Straße 5, 14195 Berlin, Germany, Tel.: +49 30 89789-338 (Fax: -103), E-mail: auhlendorff@diw.de. 


\section{$1 \quad$ Einleitung}

Die Zahl der Arbeitslosen belief sich zu Beginn des Jahres 2003 auf mehr als 4 Millionen. Untersucht man Arbeitslosigkeit in der individuellen Längsschnittperspektive, zeigt sich dass sie für die einen nur ein kurzer Zwischenstopp im Erwerbsleben ist, während sie für andere zum Dauerzustand wird. Inwiefern lassen sich die unterschiedlichen Längen individueller Arbeitslosigkeit durch beobachtbare Merkmale der arbeitslosen Akteure erklären und welche Rolle spielen in diesem Zusammenhang Persönlichkeitseigenschaften und soziale Ressourcen? Diese Fragen sind Gegenstand der vorliegenden Arbeit.

Betrachtet werden die Übergänge aus der Arbeitslosigkeit in eine Erwerbstätigkeit. Es ist dabei festzustellen, dass in West- und Ostdeutschland die das Humankapital beschreibenden Faktoren die größte Erklärungskraft haben, sich die Anwesenheit kleiner Kinder vor allem für Frauen negativ auf die Wiederbeschäftigungswahrscheinlichkeit auswirkt und ein Einfluss der Arbeitslosenquote nur im Westen beobachtbar ist. Aber auch die Persönlichkeitseigenschaften und das soziale Kapital haben Einfluss auf die Dauer der Arbeitslosigkeit: Im Westen nehmen internal kontrollierte sowie mobilitätsbereite Personen schneller eine Beschäftigung auf, während sich im Osten ein politisches oder ehrenamtliches Engagement positiv auswirkt.

Die Untersuchungseinheiten der Analyse sind einzelne Arbeitslosigkeitsepisoden. Datengrundlage ist das sozioökonomische Panel (SOEP), der betrachtete Zeitraum umfasst die Jahre von 1994 bis 2000.

Im folgenden zweiten Abschnitt dieser Arbeit wird der theoretische Rahmen kurz dargestellt, auf dem die im dritten Kapitel unterstellten Wirkungsbeziehungen aufbauen. Auf das Modell und die Daten, mit denen diese Wirkungsbeziehungen empirisch untersucht werden, wird im vierten Abschnitt eingegangen. Die Ergebnisse werden im fünften Kapitel präsentiert und die Arbeit im letzten Abschnitt noch einmal zusammengefasst.

\section{Theoretischer Rahmen}

Im Mittelpunkt der Analyse stehen Matching-Prozesse zwischen Arbeitgebern, die Arbeit nachfragen, und Erwerbspersonen, die Arbeit anbieten. Ein Arbeitsmarktmatch und ein sich anschließendes Beschäftigungsverhältnis ist eine soziale Austauschbeziehung. Tauschobjekte in solchen Beziehungen sind Kontrollrechte über Ressourcen, also materielle und immaterielle Güter, Fähigkeiten, Eigenschaften, Handlungen oder bestimmte Ereignisse. Ein Akteur kann Kontrolle über eine Ressource ausüben und Interesse an einer Ressource haben. (Coleman 1990, Esser 1999)

Im Falle eines Beschäftigungsverhältnisses überträgt der Arbeitnehmer das Kontrollrecht über einen bestimmten zeitlichen und sachlichen Bereich seiner Handlungen auf den Arbeitgeber und erhält dafür das Kontrollrecht über materielle Ressourcen seines Tauschpartners, eine Lohnzahlung. Voraussetzung für das Zustandekommen eines Tausches ist das wechselseitige Interesse an den vom potentiellen Tauschpartner kontrollierten Ressourcen. Dieses Interesse muss jeweils größer 
sein als das Interesse an den eigenen Ressourcen. Die hier betrachteten Matchingprozesse finden auf einem kompetitiven Markt statt, dem Arbeitsmarkt: Die Chance eines Arbeitsanbieters, einen Arbeitsplatz zu finden, hängt auch von den Ressourcen potentieller Mitbewerber ab (Coleman 1990,1991).

In Abbildung 2.1 ist diese Situation beispielhaft für zwei Arbeitsanbieter und einen Arbeitsnachfrager dargestellt. Die Akteure haben jeweils ein Interesse an den eigenen Ressourcen und an denen des Gegenübers. Sucht der Arbeitgeber nach nur einem Beschäftigten, konkurrieren beide Erwerbspersonen miteinander. Der Arbeitgeber wägt seine Interessen ab und entscheidet sich für die für ihn günstigste Tauschbeziehung. Auch die Konkurrenz verschiedener Arbeitgeber um eine Erwerbsperson ist denkbar. Da in dieser Untersuchung aber arbeitslos gemeldete Erwerbspersonen betrachtet werden, also Arbeitskräfte, die zumindest kurzfristig keinen Tauschpartner gefunden haben, ist die Betrachtung einer Konkurrenzsituation auf Seiten der Arbeitssuchenden ausreichend. Dies schließt nicht aus, dass die Ressourcen eines potentiellen Arbeitgebers mit Alternativen außerhalb der Erwerbstätigkeit oder mit dem Verbleib in der Arbeitslosigkeit konkurrieren.

Abbildung 2.1: Wechselseitiges Interesse an Ressourcen zwischen Tauschpartnern auf dem Arbeitsmarkt bei Konkurrenz zwischen Arbeitsanbietern

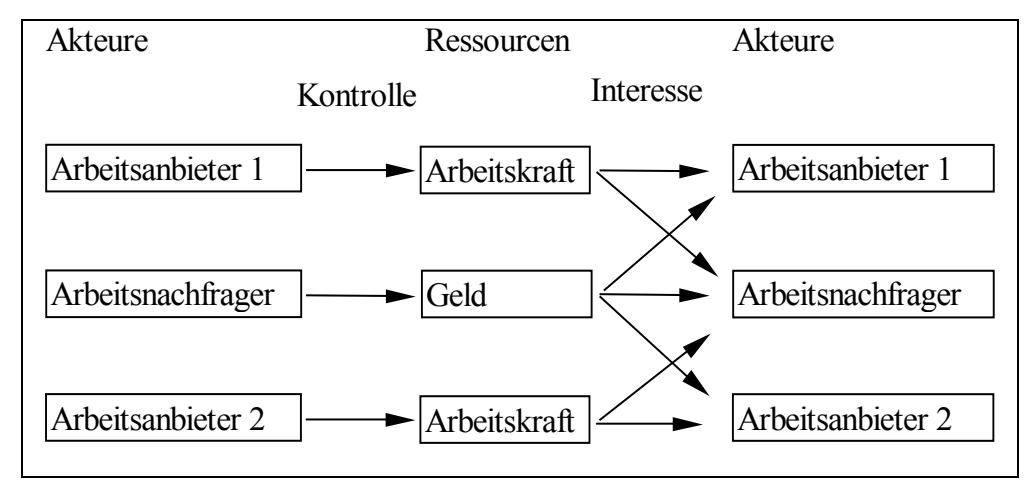

Des weiteren wird von nutzenmaximierenden Akteuren im Sinne der Rational Choice Theorie ausgegangen. Ein Individuum handelt insofern rational, als dass es diejenige Handlungsalternative aus der Gruppe der möglichen Handlungsoptionen wählt, die ihren Nutzen maximiert (Little 1991, Lindenberg 1985). Diese rationale Wahl einer Handlungsalternative wird für Arbeitslose bei der Suche nach einem Tauschpartner u.a. davon abhängen, wie hoch die Informationskosten und die Ortsgebundenheit sind, wie die Erfolgsaussichten eigener Anstrengungen eingeschätzt werden und welche Bedeutung der berufliche Erfolg für die eigene Zufriedenheit hat. Es ist daher sinnvoll, entsprechende Variablen zu berücksichtigen.

\section{Hypothesen}

Die abhängige Variable der vorliegenden Analyse ist die Wahrscheinlichkeit des Übergangs von der Arbeitslosigkeit in eine Erwerbstätigkeit. Die im folgenden betrachteten Einflussfaktoren lassen sich in vier Kategorien unterteilen. Die erste Kategorie umfasst die Umweltbedingungen, die relativ 
unabhängig von den Akteuren sind. Hierzu zählen in dieser Untersuchung die regionale Arbeitslosenquote und die Kohortenzugehörigkeit, also das Jahr, in dem die Arbeitslosigkeit beginnt. Durch entsprechende periodische Effekte werden konjunkturelle Einflüsse erfasst, auf eine Hypothesenbildung wird verzichtet.

Die zweite Kategorie umfasst die Ressourcen der arbeitslosen Erwerbspersonen in Form von beobachtbaren Eigenschaften und Fähigkeiten, die ein Interesse bei möglichen Arbeitgebern hervorrufen können und somit die Entscheidung der Nachfrager auf dem Arbeitsmarkt beeinflussen. Über diese Faktoren konkurrieren die Arbeitssuchenden miteinander.

Die Entscheidung, einen Arbeitsplatz anzunehmen bzw. die Intensität, mit der nach einem Tauschpartner gesucht wird, hängt von der finanziellen und familiären Situation der arbeitslosen Person ab. Die diese Situation erfassenden Variablen bilden die dritte Kategorie, sie beschreiben die Konkurrenzsituation der verschiedenen Zeitverwendungen auf der Ebene des einzelnen Akteurs.

In der vierten Kategorie sind die mit der Person verknüpften Faktoren zusammengefasst, die weder das Interesse eines möglichen Tauschpartners beeinflussen noch alternative Handlungsmöglichkeiten oder Einkommensquellen darstellen. Es wird also keine Konkurrenzsituation beschrieben, sondern für das Verhalten auf dem Arbeitsmarkt relevante Persönlichkeitsmerkmale und soziale Ressourcen. Derartige Einflussfaktoren werden in vergleichbaren empirischen Untersuchungen bisher i.d.R. nicht berücksichtigt (siehe bspw. Licht und Steiner 1991, Gilberg et al. 1999, Bender et al. 2000 sowie Kaiser und Siedler 2001). In Abbildung 3.1 sind die Variablen und ihre erwarteten Wirkungsrichtungen zusammenfassend dargestellt, die Einflussfaktoren der Kategorien 2 bis 4 in sind jeweils einem Block abgebildet.

Abbildung 3.1: Zusammenfassende Darstellung der betrachteten Variablen und ihrer vorhergesagten Wirkungsrichtungen

\begin{tabular}{|ll|}
\hline Ausbildungsdauer & $(+)$ \\
bisherige Erwerbsdauer & $(+)$ \\
vorherige Erwerbstätigkeit & $(+)$ \\
bisherige AL-Dauer & $(-)$ \\
Alter & $(-)$ \\
Ausländer & $(-)$ \\
Frau & $(-)$ \\
\hline \multicolumn{2}{|c|}{ Frau (-) } \\
Kinder im HH & $(-)$ \\
Kleinkinder im HH & $(-)$ \\
Einkommen des Ehepartners & $(-)$ \\
Unterstützungsanspruch & $(-)$ \\
& \\
\hline $\begin{array}{l}\text { Soziales Engagement } \\
\text { internale Kontrolleinstellung }\end{array}$ \\
Bedeutung des berufl. Erfolgs \\
Mobilitätsbereitschaft \\
\hline
\end{tabular}

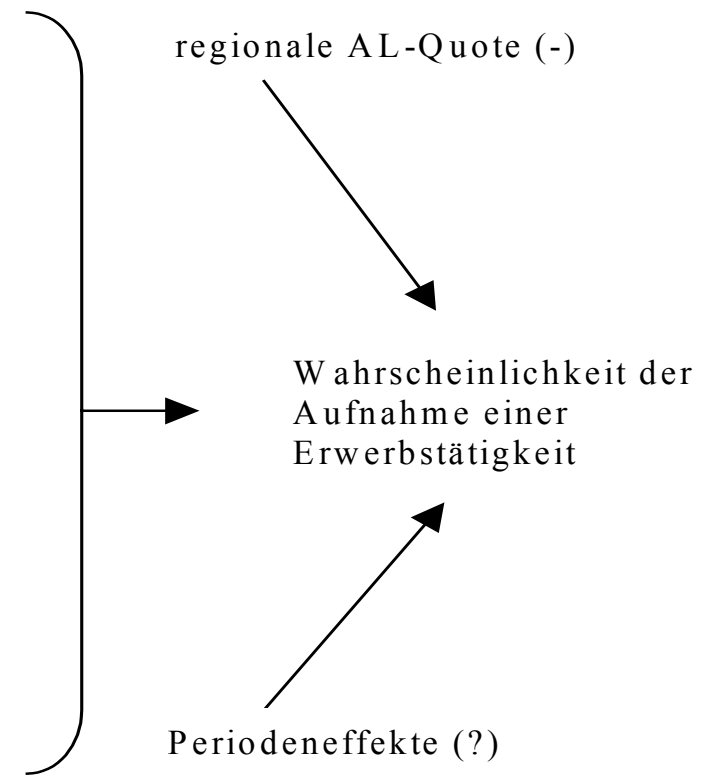


Im folgenden gehe ich auf die einzelnen Einflussfaktoren und die jeweils zugrunde liegenden theoretischen Überlegungen ein und formuliere entsprechende Hypothesen.

\section{Arbeitsmarktlage}

Die Chance einer arbeitslosen Erwerbsperson, eine Beschäftigung aufzunehmen, wird von der Konkurrenzsituation auf dem Arbeitsmarkt beeinflusst, die sich durch die regionale Arbeitslosenquote beschreiben lässt ${ }^{1}$. Die erste Hypothese lautet:

Hypothese 1: Je höher die regionale Arbeitslosenquote ist, desto geringer ist die Wahrscheinlichkeit, dass eine arbeitslose Erwerbsperson eine Erwerbstätigkeit aufnimmt.

\section{Humankapitaltheorie}

Die Bildungsinvestitionen und somit der Humankapitalstock bedingen die Verdienst- und Arbeitsmarktchancen der Erwerbspersonen. Das allgemeine Humankapital, zu dem sowohl die Schul- und Hochschulausbildung als auch berufsfachliche Ausbildungen gehören, steigert die Produktivität in verschiedenen Unternehmen in gleichem Umfang (Mincer 1974, Becker 1964). Jeder Akteur hat in diesem Sinne einen höchsten allgemeinen Bildungsabschluss, wobei die Höhe durch die für den Abschluss vorgesehene Ausbildungsdauer ausgedrückt wird. Zusätzlich zählt die bisherige Berufserfahrung einer Erwerbsperson zumindest teilweise zum allgemeinen Humankapital. Thurow beschreibt in seinem job-competition-Modell das Arbeitsangebot als eine Schlange, an deren Spitze Personen mit der besten allgemeinen Ausbildung und an deren Ende diejenigen mit der schlechtesten Ausbildung stehen. Diese relative Position in der Schlange des Arbeitsangebotes ist entscheidend für die Beschäftigungs- und Verdienstmöglichkeiten einer Erwerbsperson (Thurow 1974). Die Beschäftigungsmöglichkeiten hängen demnach von der Relation der eigenen Qualifikationen zu denen der Konkurrenten ab. Arbeitslose Personen mit höherem allgemeinem Humankapitalstock haben also größere Chancen auf die Wiederaufnahme einer Erwerbstätigkeit. Die Hypothesen lauten²:

Hypothese 2: Je länger die allgemeine Ausbildungsdauer einer arbeitslosen Erwerbsperson war, desto größer ist die Wahrscheinlichkeit der Aufnahme einer Erwerbstätigkeit.

Hypothese 3: Je länger die bisherige Erwerbstätigkeit einer arbeitslosen Erwerbsperson war, desto größer ist die Wahrscheinlichkeit der Aufnahme einer Erwerbstätigkeit.

Hypothese 4: War die arbeitslose Erwerbsperson in der Periode vor dem Eintritt in die Arbeitslosigkeit erwerbstätig, ist die Wahrscheinlichkeit der Aufnahme einer Erwerbstätigkeit größer als wenn dies nicht Fall war .

\footnotetext{
${ }^{1}$ Die hier verwendete Quote ist definiert als der (prozentuale) Anteil der arbeitslos gemeldeten Personen an den zivilen Erwerbspersonen

${ }^{2}$ Für die Dimension der Berufserfahrung wurden zwei Hypothesen gebildet. Die Hypothese 3 bezieht sich auf die bisherige, kumulierte Dauer der Erwerbstätigkeit des arbeitslosen Akteurs, unabhängig von der Zeit, die seit dieser Erwerbstätigkeit vergangen ist, während die Bedeutung der Aktualität der letzten Erwerbstätigkeit Gegenstand der Hypothese 4 ist.
} 
Humankapital verliert im Zeitverlauf an Wert. Personen vergessen das Erlernte oder das Wissen kann aufgrund technologischer Neuerungen nicht mehr in vollem Umfang Anwendung finden, es ist nicht mehr „up to date“. Ich vermute, dass der Effekt des „Vergessens“ dann besonders stark greift, wenn eine Erwerbsperson ihr Humankapital nicht verwendet, also in Phasen der Arbeitslosigkeit. Zusätzlich können potentielle Arbeitgeber die bisherige Arbeitslosigkeit einer Erwerbsperson als negatives Signal bezüglich ihrer Produktivität ansehen. Für die sogenannte „lagged duration dependence“, also den Einfluss vorhergehender Arbeitslosigkeitsdauer ${ }^{1}$ (Heckman und Borjas 1980), ergibt sich die Hypothese:

Hypothese 5: Je länger sich eine Erwerbsperson vor der aktuellen Phase der Arbeitslosigkeit in dem Zustand der Arbeitslosigkeit befand, desto geringer ist die Wahrscheinlichkeit der Aufnahme einer Erwerbstätigkeit.

Je länger ein Arbeitsverhältnis voraussichtlich andauert, desto größer sind für beide Vertragspartner die Anreize, in betriebsspezifisches Humankapital, durch welches die Produktivität im jeweiligen Betrieb erhöht wird, zu investieren. Bei älteren Arbeitslosen ist der Zeithorizont begrenzt, mit zunehmendem Alter sinkt die maximal mögliche Amortisationsdauer der Investitionen. Hinzu kommt, dass bei älteren Erwerbpersonen der Abschluss der höchsten formalen Ausbildung länger zurück liegt als bei jüngeren Erwerbspersonen und jüngere Arbeitslose aufgrund der größeren Aktualität ihrer Ausbildung einen Wettbewerbsvorteil haben.

Hypothese 6: Je älter die arbeitslose Erwerbsperson ist, desto geringer ist die Wahrscheinlichkeit der Aufnahme einer Erwerbstätigkeit.

Personen, die keine deutsche Staatsbürgerschaft aufweisen, haben tendenziell größere Verständigungsprobleme in einem neuen Arbeitsumfeld als deutsche Erwerbspersonen. Die notwendigen innerbetrieblichen Ausbildungsvorgänge sind für diese Personengruppe aufwendiger. Außerdem sind deutsche und ausländische Bildungsabschlüsse nicht immer direkt miteinander vergleichbar, es besteht zusätzliche Unsicherheit.

Hypothese 7: Ausländische Arbeitslose nehmen mit einer geringeren Wahrscheinlichkeit eine Erwerbstätigkeit auf als deutsche Arbeitslose.

Gehen potentielle Arbeitgeber bei weiblichen Bewerbern von einem vergleichsweise instabilen Beschäftigungsverhalten aus, so sinkt bei dieser Gruppe der Erwartungswert der Amortisationsdauer für Investitionen in das spezifische Humankapital.

Hypothese 8: Weibliche Arbeitslose nehmen mit einer geringeren Wahrscheinlichkeit eine Erwerbstätigkeit auf als männliche Arbeitslose. ${ }^{1}$ Der Einfluss der aktuellen Episodenlänge wird nicht untersucht, da ein Überprüfung in dem von mir
gewählten Regressionsmodell nicht möglich ist. 


\section{Familialer Kontext}

Neben der Erwerbsarbeit und der Freizeit kann ein Individuum seine Zeit für Hausarbeit aufwenden. Hierbei werden Haushaltsgüter durch den Einsatz von Zeit und Marktgütern hergestellt (Zimmermann 1985). Zu der Haushaltsarbeit zählen u.a. das Zubereiten von Nahrung und die Reinigung des Haushaltes, aber auch die Betreuung und Erziehung von Kindern. Eine Erwerbsperson wird im Optimum die Grenznutzen der drei möglichen zeitlichen Verwendungen in ein Gleichgewicht bringen. Die Haushaltsproduktivität ist dann besonders groß, wenn Kinder im Haushalt leben. Mit dieser gestiegenen Haushaltsproduktivität steigen die Opportunitätskosten beider alternativer Zeitverwendungen. Je jünger die Kinder im Haushalt sind, desto größer ist die Haushaltsproduktivität, da jüngere Kinder eine zeitlich umfangreichere Betreuung benötigen.

Der Nutzengewinn durch eine Arbeitsaufnahme hängt von der Existenz weiterer Einkommensquellen ab. Die Annahme des positiven, aber abnehmenden Grenznutzens impliziert, dass der Nutzengewinn durch eine Arbeitsaufnahme bei jedem Individuum dann höher ist, wenn keine weiteren Einkommensquellen existieren. Eine solche mögliche Einkommensquelle ist das Einkommen eines Ehepartners aus aktueller oder früherer Erwerbstätigkeit, also der Bezug von Lohn- oder Rentenzahlungen (Andreß und Strengmann-Kuhn 1997).

Ich vermute, dass Männer in stärkerem Maße dazu neigen, eine Erwerbstätigkeit aufzunehmen als Frauen. Mögliche Begründungen hierfür sind komparative Kostenvorteile bei der Betreuung vor allem kleiner Kinder (Becker 1996) sowie normative Vorstellungen bezüglich der geschlechtlichen Arbeitsteilung.

Hypothese 9: Je mehr Kinder im Haushalt eines Erwerbslosen leben, desto geringer ist die Wahrscheinlichkeit, eine Erwerbstätigkeit aufzunehmen.

Hypothese 10: Die Wahrscheinlichkeit der Aufnahme einer Erwerbstätigkeit einer arbeitslosen Erwerbsperson ist bei im Haushalt lebenden jüngeren Kindern geringer als bei im Haushalt lebenden älteren Kindern.

Hypothese 11: Arbeitslose Personen, deren Ehepartner voll erwerbstätig sind oder Renteneinkommen beziehen, nehmen mit einer geringeren Wahrscheinlichkeit eine Erwerbstätigkeit auf als arbeitslose Personen, bei denen dies nicht der Fall ist.

Hypothese 12: Sowohl die Anzahl von Kindern im Haushalt als auch die Existenz eines Einkommens eines Ehepartners wirken sich bei Frauen in stärkerem Maße negativ auf die Wahrscheinlichkeit der Aufnahme einer Erwerbstätigkeit aus als bei Männern.

\section{Arbeitslosenunterstützung}

Erhält eine arbeitslose Person eine finanzielle Arbeitslosenunterstützung, so verfügt sie über eine Einkommensquelle, für die keine zeitgleiche Gegenleistung $\mathrm{zu}$ erbringen ist. Die Bezugsberechtigung endet, wenn eine neue Erwerbstätigkeit aufgenommen wird. Bezieht ein Akteur Unterstützungszahlungen, wird der Anspruchslohn höher sein als bei Abwesenheit von 
Ersatzleistungen, was die Akzeptanzwahrscheinlichkeit von Arbeitsplatzangeboten negativ beeinflusst.

Hypothese 13: Hat die arbeitslose Erwerbsperson Anspruch auf die Auszahlung von Lohnersatzleistungen, sinkt die Wahrscheinlichkeit der Aufnahme einer Erwerbstätigkeit.

\section{Soziale Kontakte}

Werden Akteuren aufgrund von sozialen Beziehungen Handlungen ermöglicht bzw. werden mögliche Handlungen vereinfacht, so spricht man von sozialem Kapital. Soziales Kapital ist also, wie Humankapital oder ökonomisches Kapital, produktiv (Coleman 1990). Granovetter unterscheidet soziale Beziehungen nach der Stärke der interpersonalen Verbindung. Diese „strength of a tie“ besteht aus einer Kombination von Zeit, emotionaler Intensität, Intimität und reziproker Dienste. Weak ties können als Brücken zwischen sozialen Gruppen fungieren, während dies für den Fall der strong ties sehr unwahrscheinlich ist. Über die weak ties sind indirekte Kontakte zu Personen außerhalb des eigenen Bekanntenkreises möglich, sie können also wichtige Informationsquellen darstellen (Granovetter 1973, 1974). Beide Arten von sozialen Verbindungen können für den Übergang in eine Erwerbstätigkeit relevant sein, die weak ties durch den Zugang zu neuen Informationen und die strong ties durch die Inanspruchnahme von direkter Hilfe. Zusammen bilden sie das für die Beschäftigungssuche relevante soziale Kapital eines Akteurs.

Personen, die sich in ihrer Freizeit ehrenamtlich in Vereinen, Verbänden oder sozialen Diensten betätigen oder sich politisch engagieren, werden relativ viele soziale Kontakte haben, die über die Verwandtschaft, die Nachbarschaft und enge Freunde hinausgehen. Zu diesen Kontakten können sowohl weak als auch strong ties zählen, die weak ties dürften aber dominieren. Die Wahrscheinlichkeit der Erwerbstätigkeit dürfte daher bei Personen mit hohem sozialen Engagement relativ groß $\operatorname{sein}^{1}$.

Hypothese 14: Je häufiger sich erwerbslose Personen in ihrer Freizeit ehrenamtlich in Vereinen, Verbänden oder sozialen Diensten betätigen oder sich politisch engagieren, desto größer ist die Wahrscheinlichkeit der Aufnahme einer Erwerbstätigkeit.

\section{Kontrolleinstellungen}

Personen unterscheiden sich in ihrer Einschätzung, inwieweit sie dazu in der Lage sind, durch eigenes Handeln gewünschte Folgen $\mathrm{zu}$ verursachen. Diese Einschätzungen werden als Kontrolleinstellungen bezeichnet, wobei das grundlegende Konzept auf Rotter zurück geht (Nolte et al. 1997). Kontrolleinstellungen sind generalisierte Erwartungen. Weist eine Person eine internale Kontrolleinstellung auf, dann wird sie als Ursache bestimmter Handlungsfolgen in ihrem Leben vor

\footnotetext{
${ }^{1}$ Hinzu kommt die Überlegung, dass innerhalb starker sozialer Vernetzungen der Konformitätsdruck und der Grad sozialer Kontrolle dafür sorgen, dass erwerbslose Personen ihre Suchanstrengungen intensivieren (Freitag 2000)
} 
allem die eigene Person sehen. Bei externaler Kontrolleinstellung werden Faktoren außerhalb der eigenen Person als ursächlich wahrgenommen (Fischer und Wiswede 2002, Heckhausen 1989).

Personen, die eine internale Kontrolleinstellung aufweisen, verfügen vermutlich über eine starke Sebstwirksamkeitsüberzeugung sowie über eine ausgeprägte Ziel- und Planungsorientierung. Sie sind davon überzeugt, durch eigenes Handeln die Ereignisse in ihrem Leben beeinflussen, den Lebensweg also selbst (mit)bestimmen zu können. Dies bedeutet, dass „internal kontrollierte“ Akteure einer Handlungsalternative, die eigene Aktivitäten zur Zielerreichung impliziert, höhere Erwartungswerte zuordnen. Die Wahrscheinlichkeit, dass sie diese Handlungsalternative wählen, ist höher als bei andersartiger Kontrolleinstellung. Wendet man diese Überlegungen auf das Verhalten arbeitsloser Erwerbspersonen an, erscheint den Akteuren die Handlungsalternative „eigene Suche nach einem Tauschpartner" dann besonders erfolgsversprechend, wenn sie eine internale Kontrolleinstellung aufweisen.

Hypothese 15: Arbeitslose Personen, die der Meinung sind, die Folgen ihres Handeln weitgehend selbst bestimmen zu können, nehmen mit einer größeren Wahrscheinlichkeit eine Erwerbstätigkeit auf als solche, die nicht dieser Meinung sind.

\section{Bedeutung des beruflichen Erfolges}

Akteure, denen der berufliche Erfolg für ihre Zufriedenheit im Leben von großer Bedeutung ist, verbinden mit der Aufnahme einer Erwerbstätigkeit einen relativ hohen Nutzen. Dies wird sich in der Wahl einer Handlungsalternative positiv auf die Optionen „Annahme eines Arbeitsplatzangebotes“ und „eigene Suche nach einem Arbeitsplatz“ auswirken.

Hypothese 16: Arbeitslose Personen, für deren Lebenszufriedenheit der berufliche Erfolg eine bedeutende Rolle spielt, nehmen mit einer größeren Wahrscheinlichkeit eine Erwerbstätigkeit auf als solche, bei denen dies nicht der Fall ist.

\section{Mobilitätsbereitschaft}

Vergleicht man die Situation einer zur Mobilität bereiten mit der einer relativ immobilen Erwerbsperson, so kann man davon ausgehen, dass die mobile Person ihre Arbeitskraft auf einem größeren Markt anbietet, dass ihr also mehr potentielle Tauschpartner zur Verfügung stehen als einer immobilen Person. Diese größere Auswahl dürfte sich positiv auf die Beschäftigungschancen auswirken:

Hypothese 17: Arbeitslose Personen, die bereit sind, aus beruflichen Gründen den Wohnort zu wechseln, nehmen mit einer größeren Wahrscheinlichkeit eine Erwerbstätigkeit auf als solche, die dazu nicht bereit sind.

\section{Getrennte Betrachtung Ost- und Westdeutschlands}

Es werden getrennte Analysen für die neuen und die alten Bundesländer durchgeführt, da sich die Situationen auf den Arbeitsmärkten dieser beiden Teile Deutschlands sowie ihre wirtschaftlichen und gesellschaftlichen Rahmenbedingungen deutlich voneinander unterscheiden. Dazu zählen unter 
anderem divergierende sozialpolitische Interventionen, unterschiedliche wirtschaftliche Produktionsstrukturen sowie unterschiedliche strukturelle Probleme auf den Arbeitsmärkten, die sich nicht nur in den Arbeitslosenquoten widerspiegeln.

\section{Daten und Methoden}

\subsection{Das sozioökonomische Panel}

Die Datengrundlage meiner Analyse bildet das sozioökonomische Panel (SOEP). Es handelt sich um eine jährliche Wiederholungsbefragung privater Haushalte, die seit 1984 in Westdeutschland durchgeführt wird und 1990 auf das Gebiet der ehemaligen DDR ausgeweitet wurde. Die Fragen zum Haushalt werden vom Haushaltsvorstand beantwortet. Des Weiteren beantworten alle Haushaltsmitglieder, die zum Befragungszeitpunkt mindestens 16 Jahre alt sind, einen gesonderten Personalfragebogen. Es werden sowohl Ereignisse des Vorjahres wie bspw. der Erwerbsverlauf retrospektiv erfragt als auch Fragen zu der gegenwärtigen Situation wie bspw. zu der Existenz von Kindern im Haushalt oder zu Meinungen und Einstellungen gestellt. Neben wiederkehrenden Längsschnittindikatoren gibt es wellenspezifische Fragen zu bestimmten Themenbereichen. (siehe bspw. Schupp und Wagner 2002)

Das SOEP begann 1984 und wurde 1990 um eine Stichprobe erweitert, bei der die Grundgesamtheit aus Personen privater Haushalte bestand, deren Haushaltsvorstand in diesem Jahr Staatsbürger der DDR war. 1994, 1998 und 2000 kamen weitere Stichproben hinzu, die in meiner Analyse nicht berücksichtigt werden. Die von mir berücksichtigten Teilstichproben umfassten im Jahre 199412.946 befragte Personen, 2000 wurden 11.310 Personen interviewt ${ }^{1}$.

\subsection{Untersuchungszeitraum und Untersuchungseinheiten}

Berücksichtigt werden in der Analyse Erwerbspersonen, die sich $\mathrm{zu}$ Beginn einer Arbeitslosigkeitsepisode im Alter von 16 bis 59 Jahren befinden und beim Arbeitsamt arbeitslos gemeldet sind. Betrachtet wird der Zeitraum von 1994 bis 2000. Die untere Grenze dieser Zeitspanne ergibt sich aus der Tatsache, dass einige der hier berücksichtigten unabhängigen Variablen bei den vorhergehenden Befragungswellen nicht erhoben wurden.

Eine Arbeitslosigkeitsepisode stellt eine Untersuchungseinheit dar. Insgesamt liegen 5.196 Arbeitslosigkeitsepisoden für meinen Untersuchungszeitraum vor. Hiervon beginnen 649 vor dem Januar 1994. Bei weiteren 513 Fällen hat kein Interview der Befragungswellen 1994 bis 1999 vor Beginn der Arbeitslosigkeitsepisode stattgefunden. Diese Daten werden aus der Analyse ausgeschlossen, da es ist für die Untersuchung entscheidend ist, dass bestimmte unabhängige Variablen vor dem Beginn einer Arbeitslosigkeitsepisode aufgenommen werden. Dies ist für alle Variablen relevant, bei denen Rückwirkungen der Arbeitslosigkeitsdauer zu erwarten sind. Diese

1 Es gab sowohl Abgänge als auch Zugänge. Hinzu kommen bspw. in die befragten Haushalte neu eingetretene Personen oder Familienmitglieder, die das 16. Lebensjahr erreicht haben. Außerdem können Personen in einigen Jahren Befragungslücken aufweisen. 
Gefahr besteht v.a. bei der Kontrolleinstellung, aber auch bei der Bedeutung des beruflichen Erfolges, der Mobilitätsbereitschaft und dem sozialen Engagement ${ }^{1}$. Beginnt somit bspw. eine Episode im Februar 1995 und fand für die betroffene Person im Jahre 1994 kein und im Jahre 1995 erst im März ein Interview statt, wird diese Episode nicht in die Stichprobe aufgenommen. Des weiteren werden alle „regulär“ linkszensierten Daten nicht berücksichtigt und nur solche Episoden betrachtet, die innerhalb von seit dem Januar 1992 kontinuierlich dokumentierten Erwerbsbiographien eingetreten sind. Dies bedeutet nicht, dass die Teilnehmer in jedem Jahr an der Befragung teilgenommen haben, wohl aber dass die Erwerbsbiographie für eventuelle Lücken nacherhoben wurde. Letztendlich reduziert sich die Fallzahl somit auf $n=3143$. Diese 3143 Arbeitslosigkeitsepisoden verteilen sich auf 2026 Personen, woraus sich eine durchschnittliche Anzahl von 1,6 Arbeitslosigkeitsepisoden pro in meiner Stichprobe vertretenen Person ergibt.

Wie Licht und Steiner (1991) verstehe ich unter erwerbstätigen Personen solche, die voll- oder teilzeitbeschäftigt sind, sich in Kurzarbeit befinden, geringfügig erwerbstätig und nicht gleichzeitig arbeitslos gemeldet sind oder sich in betrieblicher Ausbildung, Fortbildung oder Umschulung befinden. Personen, die sich in sogenannten Arbeitsbeschaffungsmaßnahmen (ABM) befinden, zählen zu der Gruppe der Voll- oder Teilzeitbeschäftigen und sind nach meiner Einteilung dementsprechend ebenfalls erwerbstätig. Arbeitslos sind diejenigen Personen, die beim Arbeitsamt als arbeitslos gemeldet sind, alle anderen Zustände werden in der Gruppe der Nichterwerbstätigkeit zusammengefasst (siehe auch Anhang A).

Einen Überblick über die Anzahl der berücksichtigten Arbeitslosigkeitsphasen sowie der Folgezustände liefert die Tabelle 4.1.

Tabelle 4.1: Anzahl der Arbeitslosigkeitsepisoden und ihrer Anschlusszustände getrennt nach Ost- und Westdeutschland

\begin{tabular}{|l|cl|cl|cl|}
\hline & \multicolumn{2}{|c|}{ West } & \multicolumn{2}{|c|}{ Ost } & \multicolumn{2}{|c|}{ Gesamt } \\
\hline Episodenanzahl & $1508(100)$ & 1635 & $(100)$ & 3143 & $(100)$ \\
Anschlusszustand: & & & & & & \\
Erwerbstätigkeit & 873 & $(57.9)$ & 1086 & $(66.4)$ & 1959 & $(62.3)$ \\
Nichterwerbstätigkeit & 320 & $(21.2)$ & 235 & $(14.4)$ & 555 & $(17.7)$ \\
Rechts zensiert & 315 & $(20.9)$ & 314 & $(19.2)$ & 629 & $(20.0)$ \\
\hline
\end{tabular}

In beiden Landesteilen ist die Erwerbstätigkeit der häufigste auf eine Arbeitslosigkeit folgende Anschlusszustand. Dieser Anteil liegt im Osten Deutschlands über dem im Westen, wo sich vergleichsweise häufiger eine Nichterwerbstätigkeit anschließt.

\footnotetext{
${ }^{1}$ Bei den übrigen Variablen sind mögliche Rückwirkungen zwar ebenfalls denkbar, aber unproblematisch, da diese nacherhoben werden und somit i.d.R. die jeweilige Ausprägung zu Beginn der Episode vorliegt. Der Interviewtermin vor dem Episodenbeginn ist also nicht zwingend, durch die Restriktion für die Variablen der Kategorie 4 aber gegeben.
} 


\subsection{Variablenübersicht}

In Tabelle 4.2 sind die Mittelwerte und Standardabweichungen für die in meinen Analysen verwandten Variablen angegeben. Berücksichtigt werden alle gültigen Werte der jeweiligen Variable. Eine Ausnahme bildet die Variable „Mobilitätsbereitschaft“. In den Jahren 1994 und 1995 wurde ein Großteil der ausländischen Untersuchungspersonen nicht nach ihrer Mobilitätsbereitschaft befragt. Es handelt sich um 207 Fälle. Für diese Untersuchungseinheiten wird der fehlende Wert jeweils durch den Mittelwert der Mobilitätsbereitschaft aller Arbeitslosigkeitsepisoden ersetzt, die ausländischen Personen zugeordnet werden können. Eine getrennte Betrachtung der Geschlechter wird bei den Variablen vorgenommen, bei denen Interaktionseffekte mit dem Geschlecht zu erwarten sind.

Das soziale Engagement unterscheidet sich nicht zwischen den neuen und den alten Bundesländern. Es handelt sich um einen additiven Index, der sich aus der Häufigkeit ehrenamtlicher Tätigkeiten in Vereinen, Verbänden oder sozialen Diensten und der Häufigkeit der Beteiligung in Bürgerinitiativen, Parteien oder in der Kommunalpolitik zusammensetzt. Der Wertebereich reicht von 2 (kein Engagement) bis 8 (für beide Bereiche wöchentliches Engagement). Ungefähr fünfzig Prozent der Untersuchungspersonen sind der Meinung, dass sie relativ viel Kontrolle über die Ereignisse in ihrem Leben ausüben, sie weisen eine internale Kontrolleinstellung auf. Voraussetzung für eine entsprechende Kodierung ist die Zustimmung zu drei Aussagen, mit denen die Selbstwirksamkeitsüberzeugung sowie die Ziel- und Planungsorientierung der Untersuchungspersonen erfasst werden soll. Im Osten ist der Anteil derjenigen, für die der berufliche Erfolg sehr wichtig für ihre Zufriedenheit ist, höher als im Westen, während hier die befragten Personen eher bereit sind, aus beruflichen oder familiären Gründen den Wohnort zu wechseln.

Die durchschnittliche Länge der Arbeitslosigkeitsepisoden unterscheidet sich zwischen beiden Landesteilen kaum und liegt bei knapp 10 Monaten. Auf eine Beschreibung der weiteren Variablen wird verzichtet, die Werte können in Tabelle 4.2 abgelesen werden. Eine genauere Beschreibung der Operationalisierungen befindet sich in Anhang A.

\section{Tabelle 4.2 ungefähr hier}

\subsection{Ereignisanalytische Methoden}

Es liegen zwei mögliche Ereignisse vor: Austritt aus der Arbeitslosigkeit mit und Austritt aus der Arbeitslosigkeit ohne Übergang in eine Erwerbstätigkeit. Derartige Zustandswechsel lassen sich mit den Methoden der Ereignisanalyse untersuchen (Blossfeld et al. 1986). In dieser Arbeit werden die Überlebensfunktionen für beide möglichen Ereignissen sowie eine unproportionale Cox-Regression geschätzt. 


\section{Überlebensfunktionen}

Eine Survivorfunktion gibt die Überlebenswahrscheinlichkeit eines Objektes in einem bestimmten Zustand bis zum Zeitpunkt $\mathrm{t}$ an, in dem hier behandelten Fall also die Wahrscheinlichkeit, bis zum Zeitpunkt $t$ arbeitslos zu sein. Es handelt sich um eine nichtparametrische, deskriptive Methode, bei der keine bestimmte Verteilung der Ereignisse bzw. ihrer Eintrittswahrscheinlichkeiten vorausgesetzt wird. Liegen wie hier konkurrierende Risiken vor, spricht man von PseudoÜberlebensfunktionen, die hier mit der Kaplan-Meier-Methode geschätzt werden. Jeder Zeitpunkt, an dem mindestens ein Ereignis eintritt, wird in der Berechnung berücksichtigt, die vorhandenen Informationen werden also optimal ausgeschöpft. Formal entspricht dieser Schätzer folgendem Ausdruck:

$$
\widetilde{G}_{k}(t)=\prod_{l: \tau_{k, l}<t}\left(1-\frac{E_{k, l}}{R_{l}}\right)
$$

Für jeden möglichen Zielzustand $k$, also in meinem Falle für die Zustände Erwerbstätigkeit und Nichterwerbstätigkeit, wird jeder Zeitpunkt, zu dem mindestens ein Übergang stattfindet, betrachtet. Der Ausdruck $E_{k, l}$ beinhaltet die Anzahl der Ereignisse der Gruppe $k$ zum Zeitpunkt $l$. Der Ausdruck $R_{l}$ enthält die Anzahl aller Episoden, die den Zeitpunkt $l$ erreichen. In dieser Risikomenge werden also auch die Episoden berücksichtigt, die rechtszensiert sind sowie diejenigen, die einen anderen Anschlusszustand haben. (Blossfeld und Rohwer 2002)

\section{Auswahl eines geeigneten ökonometrischen Modells}

In Übergangsratenmodellen werden sogenannte Übergangs- oder Hazardraten geschätzt. Die Hazardrate $r(t)$ kann interpretiert werden als die Neigung bzw. die Wahrscheinlichkeit eines Objektes, zum Zeitpunkt $t$ vom Zustand $j$ in den Zustand $k$ zu wechseln, hier also als die bedingte Wahrscheinlichkeit, dass ein arbeitsloser Akteur zum Zeitpunkt $t$ eine Erwerbstätigkeit aufnimmt. Die Hazardraten können in Abhängigkeit der Ausprägungen verschiedener Kovariablen variieren. Es stehen verschiedene Verfahren zur Modellierung dieser Prozesse zur Verfügung.

Es lassen sich parametrische und semi-parametrische Modelle unterscheiden. Bei parametrischen Übergangsratenmodellen wird eine bestimmte Zeitabhängigkeit der Hazardrate angenommen, der funktionale Zusammenhang zwischen der Übergangsrate, den Kovariaten und der Verweildauer wird genau spezifiziert. Bei semi-parametrischen Modellen ist dies nicht der Fall, der Typ der Verweildauerabhängigkeit wird offen gelassen und lediglich der Funktionszusammenhang zwischen der Hazardrate und den Kovariablen festgelegt. Da die Verweildauerabhängigkeit in dieser Analyse nicht betrachtet werden soll, bietet sich für meine Untersuchung die Wahl eines semiparametrischen Modells an. Das üblicherweise verwendete semi-parametrische Modell ist das sogenannte Proportional-Hazard-Modell von Cox, auch Cox-Regression genannt (Yamaguchi 1991). Formal kann das Modell wie folgt ausgedrückt werden: 


$$
r(t)=h(t) \exp (A(t) \alpha)
$$

wobei $h(t)$ die Basisübergangsrate darstellt. Diese Basisübergangsrate ist unspezifiziert, wodurch die Flexibilität des Modells erreicht wird (Blossfeld et al. 1986). Der Zeilenvektor $A(t)$ enthält die Ausprägungen der Kovariablen, während der Spaltenvektor $\alpha$ die dazugehörigen Koeffizienten beinhaltet. Das Modell baut auf der Annahme proportionaler Hazardraten auf: Die Basisrate $\mathrm{h}(\mathrm{t})$ ist für alle Personen gleich und die Ausfallrisiken zweier Individuen unterscheiden sich lediglich durch einen Proportionalitätsfaktor, der unabhängig von der Verweildauer konstant bleibt (Blossfeld und Rohwer 2002).

Das proportionale Coxmodell sollte nur dann angewendet werden, wenn die Proportionalitätsannahme gerechtfertigt ist (bspw. Yamaguchi 1991 oder Blossfeld und Rohwer 2002). In dem hier verwandten Programmpaket TDA stehen zwei Möglichkeiten zur Verfügung, die Gültigkeit dieser Annahme zu testen (Pötter und Rohwer 1999). Der globale Test auf Proportionalität nach Moreau et al. ergibt für meinen Datensatz für beide Landesteile jeweils ein hochsignifikantes Ergebnis ${ }^{1}$, die Proportionalitätsannahme kann also nicht aufrechterhalten werden. Der zweite mögliche Test baut auf der Annahme auf, dass bei gegebener Proportionalität keine Interaktionseffekte der Verweildauer bzw. der logarithmierten Verweildauer mit den einzelnen Kovariablen auftreten dürften. Sind die Koeffizienten der gebildeten Interaktionsterme signifikant von Null verschieden, muss man für die jeweilige Kovariable die Proportionalitätsannahme verwerfen. Signifikante Interaktionseffekte treten für beide Landesteile bei den Variablen Kleinkinder im Haushalt weiblicher Arbeitsloser und Unterstützungsanspruch auf, im Westen kommen entsprechende Effekte für Alter, Kinder im Haushalt weiblicher Arbeitsloser und Mobilitätsbereitschaft hinzu, im Osten für die bisherige Arbeitslosigkeitsdauer, Geschlecht und Kinder im Haushalt männlicher Arbeitsloser.

Aufgrund dieser Interaktionseffekte wird statt einer proportionalen eine unproportionale CoxRegression geschätzt. Die ermittelten signifikanten Interaktionseffekte werden hierbei in das Modell mit einbezogen, wodurch die Verletzung der Proportionalitätsannahme korrigiert wird (Blossfeld und Rohwer 2002 oder Yamaguchi 1991). Die Interpretation der Effekte wird durch dieses Vorgehen erschwert, das Modell liefert aber eine akzeptable Anpassung an die vorliegenden Daten. Die Interaktionsterme sind jeweils modelliert als das Produkt der logarithmierten Verweildauer $(\ln ($ Dauer $))$ mit einer Kovariablen. Die Effekte der entsprechenden Kovariablen variieren somit über die Zeit ${ }^{2}$. Das Modell kann wie folgt geschrieben werden:

1 Die entsprechenden Werte der (Chi-Quadrat-verteilten) Teststatistiken betragen $99.72 \quad$ mit 72 Freiheitsgraden für den Westen und 101.89 mit 72 Freiheitsgraden für den Osten (Intervallgrenzen: 4,8,12 Monate).

${ }^{2}$ Der jeweilige monatliche Koeffizient kann berechnet werden durch Addition des ermittelten Koeffizienten für die entsprechende Kovariable mit dem Produkt des Interaktionseffektes und der logarithmierten Verweildauer (Yamaguchi 1991). 


$$
r(t)=h(t) \exp \left(A(t) \alpha+A_{i} * \log (\text { time }) * \alpha_{i}\right),
$$

wobei $A_{i}$ die Kovariablen beinhaltet, für die die Proportionalitätsannahme nicht gehalten werden kann und $\alpha_{i}$ die entsprechenden Interaktionseffekte mit der logarithmierten Zeit enthält.

Das betrachtete Modell beinhaltet keinen Fehlerterm, um für unbeobachtete Heterogenität zu kontrollieren. Es gibt Argumente, die für die Einbeziehung eines solchen Fehlerterms sprechen, da im Falle unberücksichtigter Heterogenität verzerrte Effektschätzungen auftreten können. Allerdings birgt auch die Einbeziehung eines Fehlerterms für sich die Gefahr verzerrter Schätzungen (Narendranathan und Stewart 1993). Es besteht die Gefahr, dass das Problem lediglich auf eine andere Ebene verschoben wird (Blossfeld und Rohwer 2002). In dieser Untersuchung wird der Einfluss von Variablen berücksichtigt, die Faktoren abbilden, welche üblicherweise als mögliche Quellen unbeobachteter Heterogenität angesehen werden: soziale Ressourcen und Persönlichkeitsmerkmale wie Motivation und Kontrolleinstellung.

\section{Ergebnisse}

In Abbildung 5.1 sind die Pseudoüberlebensfunktionen beider konkurrierenden Risiken für Westund Ostdeutschland dargestellt, wobei auf der Ordinate die Überlebensfunktion und auf der Abszisse die Anzahl der Monate abgetragen sind.

\section{Abbildung 5.1: Pseudoüberlebensfunktionen für die Übergänge in eine Erwerbstätigkeit und in eine Nichterwerbstätigkeit in West- und Ostdeutschland}
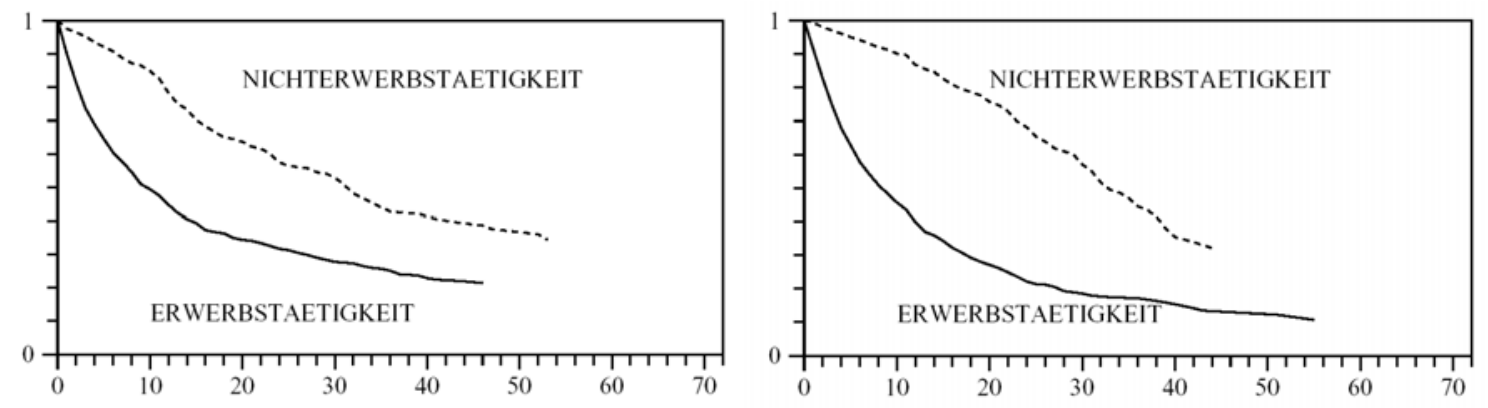

Der Verlauf der Überlebensfunktion für den Übergang in eine Erwerbstätigkeit liegt in beiden Landesteilen zu jedem Zeitpunkt unterhalb des Funktionsverlaufes für den Übergang in eine Nichterwerbstätigkeit. Der prozentuale Anteil der Personen, die sich noch im Ausgangszustand der Arbeitslosigkeit befinden, ist bei den Übergängen in die Nichterwerbstätigkeit in jedem Monat höher als bei der Aufnahme einer Beschäftigung, die Erwerbstätigkeit „zieht“ die Akteure in stärkerem Maße aus der Arbeitslosigkeit. Für den Westen liegt der Median der Pseudoüberlebensfunktion für den Übergang in eine Beschäftigung mit 9,60 Monaten über dem im Osten mit 8,40, d.h. zu diesen Zeitpunkten sind jeweils 50\% der Personen noch im Ausgangszustand der Arbeitslosigkeit. Die entsprechenden Werte liegen für die Austritte aus dem Arbeitsmarkt bei 31,29 Monaten im Westen und 32,77 Monaten im Osten. 
In den Tabellen 5.1 und 5.2 sind die Ergebnisse der unproportionalen Coxregressionen für Westund Ostdeutschland abgebildet. Ich unterscheide vier Modelle, wobei im ersten Modell der Einfluss von Kovariablen der in Abschnitt 3 beschriebenen ersten Kategorie geschätzt wird, in den weiteren Modellen kommen dann die Kovariablen der zweiten, dritten bzw. vierten Kategorie hinzu.

\section{Wirtschaftliche und gesetzliche Rahmenbedingungen}

Im Westen reduziert die Steigerung der Arbeitslosenquote um einen Prozentpunkt die Übergangsrate in eine Beschäftigung um 7.6\% ${ }^{1}$. Im Osten ist kein signifikanter Einfluss festzustellen, die Hypothese 1 kann hier nicht bestätigt werden. Die Veränderung des LogLikelihoods ist für beide Landesteile signifikant, durch Berücksichtigung der Umweltbedingungen auf dem Arbeitsmarkt wird die Modellgüte bzw. die Erklärungskraft des Modells verbessert.

\section{Kennzeichen des Arbeitsangebotes}

Die Ausbildungsdauer und eine vorherige Erwerbstätigkeit haben in beiden Landesteilen signifikant positive Auswirkungen auf die Übergangswahrscheinlichkeit in eine Erwerbstätigkeit. Im Osten weist ein Hochschulabsolvent gegenüber einer Person ohne Abschluss eine um $60 \%$ höhere Wahrscheinlichkeit der Arbeitsaufnahme auf, im Westen sind es 114\%. Eine vorherige Erwerbstätigkeit erhöht die Wahrscheinlichkeit einer Beschäftigung im Westen um 52\%, im Osten um 27\%, die Hypothesen 2 und 4 werden bestätigt. Die Dauer der bisherigen Erwerbstätigkeit hat hingegen keinen Einfluss.

Während für die bisherige Arbeitslosigkeitsdauer im Westen keine negativen Auswirkungen auf die Arbeitsmarktchancen nachweisbar sind, ist für Ostdeutschland eine differenziertere Betrachtung der Effekte notwendig. Wie Tabelle 5.2 zeigt, liegt für den ersten Monat der Arbeitslosigkeit entsprechend der Hypothese 5 ein signifikant negativer Effekt vergangener Arbeitslosigkeitsdauer vor. Dieser Effekt reduziert sich allerdings im Zeitverlauf, wie in Tabelle B.3 beispielhaft dargestellt ist. Die Abnahme des Einflusses der vorherigen Arbeitslosigkeitsdauer ist intuitiv einleuchtend, wenn man die gesamte Arbeitslosigkeitserfahrung als Summe der aktuellen und der vergangenen Arbeitslosigkeitsdauer auffasst. Mit wachsender Dauer der aktuellen Phase gewinnt diese an Gewicht, der Einfluss vorheriger Arbeitslosigkeit geht zurück.

In beiden Landesteilen nimmt entsprechend der Hypothese 6 mit zunehmendem Alter die Chance ab, eine Erwerbstätigkeit aufzunehmen. Während dieser Effekt im Osten im Zeitverlauf konstant ist, verstärkt er sich im Westen mit zunehmender Arbeitslosigkeitsdauer.

Ausländische Arbeitlose (-37\%) und Frauen (-22\%) haben im Westen eine hochsignifikant geringere Wahrscheinlichkeit der Wiederbeschäftigung. Im Osten lässt sich kein eindeutiger

\footnotetext{
${ }^{1}$ Hier und im folgenden bezieht sich die Interpretation der Effekte auf die in Modell 4 berechneten Koeffizienten.
} 
Einfluss der Nationalität belegen ${ }^{1}$. Der Nachteil der Frauen ist im ersten Monat mit einer um 47\% geringeren Übergangswahrscheinlichkeit hochsignifikant, mit der Zeit verliert das Geschlecht aber an Bedeutung. Nach einem Jahr ist dieser Effekt nur noch schwach negativ (Tabelle B.4). Die Hypothesen 7 und 8 werden weitgehend bestätigt.

Die Beobachtungen zum Humankapital decken sich weitgehend mit den Ergebnisssen anderer empirischer Untersuchungen. Es sind nicht immer die gleichen Effekte signifikant, aber es werden keine konträren signifikanten Effekte festgestellt (siehe bspw. Licht und Steiner 1991, Gilberg et al. 1999, Bender et al. 2000 sowie Kaiser und Siedler 2001). Auch in anderen Ländern lassen sich positive Auswirkungen des Humankapitals auf die Erwerbswahrscheinlichkeit beobachten (bspw. Wolbers 2000 für die Niederlande, Kaiser und Siedler 2001 für Großbritannien, und Roed und Zhang 2003 für Norwegen).

Durch die Berücksichtigung von Kovariablen, die die Attraktivität der arbeitslosen Akteure für potentielle Arbeitgeber determinieren, wird die Modellgüte signifikant verbessert. Es kommt zu einem vergleichsweise starken Anstieg des Pseudo- $\mathrm{R}^{2}$.

\section{Familiale und finanzielle Opportunitäten}

Im Westen wirkt sich die Anzahl der Kinder im Alter zwischen 5 und 14 Jahren bei weiblichen Arbeitslosen im Zeitverlauf positiv auf die Übergangswahrscheinlichkeit aus. Für die Anzahl der Kleinkinder lässt sich im ersten Monat ein hoch signifikant negativer Effekt auf die Wiederbeschäftigungswahrscheinlichkeit ausmachen, doch auch hier existiert ein positiver Interaktionseffekt mit der Zeit. Bei den Männern ist ein schwach positiver Effekt der Anzahl älterer Kinder im Haushalt zu beobachten. In den neuen Bundesländern hat die Anzahl älterer Kinder keinen Einfluss auf die Arbeitsmarktsituation der Frauen. Die Anzahl von Kleinkindern wirkt sich ähnlich wie in den alten Bundesländern aus: Es existiert zunächst ein deutlich negativer Effekt, dem ein positiver Interaktionseffekt mit der Zeit entgegenwirkt. Bei den Männern lässt sich ein schwach positiver Interaktionseffekt der Anzahl älterer Kinder mit der Zeit ausmachen.

Die Anwesenheit von Kleinkindern stellt in Deutschland also v.a. für Frauen eine zeitliche Alternative zur Erwerbsarbeit dar, während ältere Kinder arbeitslose Personen nicht von der Aufnahme einer Erwerbstätigkeit abzuhalten scheinen. Ihre Anwesenheit wirkt sich teilweise sogar positiv aus. Eine mögliche Ursache hierfür könnte die relative Selbständigkeit älterer Kinder und ein mit der Kinderanzahl wachsender finanzieller Bedarf in der Familie sein. Diese Beobachtungen widersprechen den Befunden anderer Untersuchungen nicht.

Für die Erwerbstätigkeit oder das Renteneinkommen eines Ehepartners lässt sich lediglich im Osten ein signifikanter Effekt beobachten: Männliche Arbeitslose nehmen bei der Existenz eines Partnereinkommens mit um 28\% höherer Wahrscheinlichkeit eine Erwerbstätigkeit auf. Im Bereich

\footnotetext{
${ }^{1}$ Dies kann damit zusammenhängen, dass die Anzahl ausländischer Arbeitsloser in der „Oststichprobe“ sehr gering ist.
} 
der Familie scheint die negative Anreizwirkung alternativer Einkommensquellen nicht vorhanden $\mathrm{zu}$ sein bzw. durch andere Effekte überlagert $\mathrm{zu}$ werden. Der Anspruch auf Unterstützungszahlungen wirkt sich im Westen und im Osten zu Beginn einer Arbeitslosigkeit deutlich negativ aus, dieser Effekt nimmt aber in beiden Landesteilen im Zeitverlauf ab. Die Hypothesen 10, 12 und 13 werden überwiegend bestätigt.

Insgesamt wird die Erklärungskraft des Modells durch die Einbeziehung der familiären und finanziellen Situation der Arbeitslosen sowohl in West- als auch in Ostdeutschland signifikant erhöht, was sich auch in einer Erhöhung des Pseudo- $\mathrm{R}^{2}$ ausdrückt.

\section{Soziale Ressourcen und Persönlichkeitsmerkmale}

Das soziale Engagement, das eine Dimension des sozialen Kapitals erfasst, wirkt sich in Westdeutschland nicht auf die Wiederbeschäftigungschancen der arbeitslosen Erwerbspersonen aus, im Osten wird ein schwach positiver Effekt identifiziert. Eine arbeitslose Person, die sich wöchentlich sowohl ehrenamtlich in Vereinen, Verbänden oder sozialen Diensten als auch politisch engagiert, hat gegenüber einer Person ohne soziales Engagement eine um 45\% erhöhte Wahrscheinlichkeit, eine Erwerbstätigkeit aufzunehmen. Politische und ehrenamtliche Netzwerke scheinen in den neuen Bundesländern für die Chancen im Erwerbsleben eine Rolle zu spielen, die Hypothese 14 wird hier bestätigt. Der Einfluss von sozialen Kontakten wird in anderen Untersuchungen relativ selten getestet. Die bisher angeführten vergleichbaren Arbeiten enthalten keine entsprechenden Kovariablen. Für die Niederlande hat Sprengers für männliche Arbeitslose zwischen 40 und 55 Jahren einen signifikant positiven Effekt der sozialen Ressourcen festgestellt (1992), während Barbieri et al. in einer Untersuchung verschiedener europäischer Länder keinen Effekt der Dimension des sozialen Kapitals, welches das sozial Netzwerk erfasst, ausmachen (2000). Eine Analyse des Eurobarometers 1996 ergibt, dass 31\% der in Deutschland befragten Personen angeben, ihre derzeitige Stelle über informelle Kontakte wie Freunde, Bekannte und Familienangehörige gefunden zu haben, der Durchschnitt der EU-Länder liegt sogar bei 41\% (Noll und Weick 2002). Soziale Ressourcen scheinen also eine gewisse Bedeutung auf dem Arbeitsmarkt zu haben, auch wenn in den bisherigen Analysen nicht immer signifikante Effekte ermittelt werden.

Personen, die eine internale Kontrolleinstellung aufweisen, nehmen im Westen mit einer um 17\% höheren Wahrscheinlichkeit eine Erwerbstätigkeit auf als Personen, die nicht davon überzeugt sind, die Folgen ihres Handelns weitgehend selbst bestimmen zu können. Im Osten hat die Kontrolleinstellung keine Auswirkung auf die Arbeitsmarktchancen. Die Bedeutung des beruflichen Erfolges wirkt sich sowohl in West- als auch in Ostdeutschland nicht auf die Länge der individuelle Arbeitslosigkeit aus. Personen, die bereit sind, aus beruflichen oder familiären Gründen ihren Wohnort zu wechseln, haben im Westen einen im Zeitverlauf zunehmenden Wettbewerbsvorteil. Nach einem Jahr Arbeitslosigkeit nehmen mobilitätsbereite Personen mit einer um 25\% höheren 
Wahrscheinlichkeit eine neue Beschäftigung auf. Im Osten zahlt sich die Mobilitätsbereitschaft dagegen nicht aus. Die Hypothesen 15 und 17 werden nur im Westen bestätigt.

Ebenso wie soziale Ressourcen sind auch Persönlichkeitseigenschaften selten Gegenstand von Untersuchungen individueller Arbeitslosigkeitsdauer. Nolte et al. stellen für den Einfluss der internalen Kontrolleinstellung keine signifikanten Effekte fest (1997), wobei ihre Stichprobe aus Personen besteht, die 1994 arbeitslos waren und im Rahmen des SOEP befragt wurden. Dieser Befund kann allerdings Folge eines relativ einfachen Untersuchungsdesigns sein ${ }^{1}$. Längsschnittuntersuchungen, die die Bedeutung des beruflichen Erfolges oder die Mobilitätsbereitschaft untersuchen, sind mir nicht bekannt.

Im Westen führt die Berücksichtigung der sozialen Ressourcen und Persönlichkeitseigenschaften zu einer schwach signifikanten Verbesserung der Erklärungskraft des Modells, im Osten ist der Log-Likelihood-Ratio-Test nicht signifikant. Die Existenz signifikanter Effekte in beiden Landesteilen spricht allerdings für eine Einbeziehung entsprechender Kovariablen. Zudem vermute ich, dass eine Weiterentwicklung der hier vorgestellten Messmethoden für die relevanten Persönlichkeitseigenschaften und sozialen Ressourcen zu eindeutigeren Ergebnissen führen könnten. Vergleicht man die Entwicklung der übrigen signifikanten Einflussfaktoren, so ändern sich Richtung und Signifikanz durch Berücksichtigung der Persönlichkeitseigenschaften und sozialen Ressourcen nicht, wie man bei einem Vergleich der Modelle 3 und 4 (siehe Tabellen 5.1 und 5.2) erkennen kann. Die in anderen Untersuchungen gewonnenen Erkenntnisse werden durch die hier vorgeschlagenen Modellerweiterungen also nicht in Frage gestellt, aber sinnvoll ergänzt.

\section{Zusammenfassung}

In der vorliegenden Arbeit wurde der Einfluss verschiedener Faktoren auf die individuelle Arbeitslosigkeitsdauer mittels einer unproportionalen Cox-Regression ohne Berücksichtigung unbeobachteter Heterogenität untersucht. Im Vergleich zum üblichen Vorgehen wurde hierbei auch der Einfluss von Persönlichkeitseigenschaften und sozialen Ressourcen betrachtet.

Es zeigt sich, dass vor allem die Eigenschaften, die vom Arbeitgeber beobachtbar sind, große Erklärungskraft haben. Ältere Erwerbspersonen, Frauen, Geringqualifizierte und Personen, die vor ihrer Arbeitslosigkeit nicht erwerbstätig waren, haben gegenüber ihren Konkurrenten Wettbewerbsnachteile: Die Gefahr einer längeren Arbeitslosigkeit ist für sie besonders groß. Für den Osten ist zusätzlich beobachtbar, dass es für Arbeitslose mit zunehmender vorheriger Arbeitslosigkeitserfahrung schwerer wird, aus der erneuten Arbeitslosigkeit den Weg zurück in das Berufsleben zu finden, im Westen haben Ausländer Wettbewerbsnachteile. Auch die familiale Situation hat Auswirkungen auf die individuelle Arbeitslosigkeitsdauer. Die Betreuung von Kleinkindern ist hierbei aber offenbar nur für Frauen eine zeitliche Alternative zur Erwerbsarbeit.

${ }^{1}$ Es wurde der Zustand des Folgejahres festgehalten (Arbeitslosigkeit oder Erwerbstätigkeit) und für die Kontrolleinstellung beider Gruppen kein signifikanter Unterschied beobachtet. 
Der Bezug einer Arbeitslosenunterstützung wirkt sich nur zu Beginn einer Arbeitslosigkeitsphase negativ aus.

Die Persönlichkeitsmerkmale liefern in Westdeutschland zusätzliche Erklärungskraft, hier gehen internal kontrollierte sowie zur Mobilität bereite Personen schneller in eine Beschäftigung über. Soziale Kontakte über ehrenamtliches oder politisches Engagement zahlen sich im Osten positiv aus. Die Analyse zeigt also, dass eine Berücksichtigung dieser in vergleichbaren Untersuchungen eher vernachlässigten Variablen möglich ist und interessante Einsichten liefert. Es ist zu vermuten, dass eine verstärkte Berücksichtigung und Weiterentwicklung derartiger Kovariablen und ihrer Operationalisierungen einen Beitrag zur Reduzierung der ,unbeobachtbaren“ Heterogenität der Untersuchungspersonen liefern können und sich aus den ermittelten Einflussfaktoren neue Erkenntnisse über die Überwindung individueller Arbeitslosigkeit ergeben. 
Tabelle 4.1: Mittel- bzw. Anteilswerte und Standardabweichungen der Variablen in Ostund Westdeutschland

\begin{tabular}{|c|c|c|c|c|}
\hline & \multicolumn{2}{|c|}{ West } & \multicolumn{2}{|c|}{ Ost } \\
\hline & Frauen & Männer & Frauen & Männer \\
\hline $\begin{array}{l}\text { Regionale Arbeitslosenquote im Jahr des AL-Beginns } \\
\text { (Durchschnitt in \%) }\end{array}$ & \multicolumn{2}{|c|}{$\begin{array}{l}10.01 \\
(2.31)\end{array}$} & \multicolumn{2}{|c|}{$\begin{array}{l}17.64 \\
(2.13)\end{array}$} \\
\hline Beginn der Arbeitslosigkeitsphase 1994 & \multicolumn{2}{|c|}{0.13} & \multicolumn{2}{|c|}{0.15} \\
\hline Beginn der Arbeitslosigkeitsphase 1995 & \multicolumn{2}{|c|}{0.19} & \multicolumn{2}{|c|}{0.20} \\
\hline Beginn der Arbeitslosigkeitsphase 1996 & \multicolumn{2}{|c|}{0.19} & \multicolumn{2}{|c|}{0.21} \\
\hline Beginn der Arbeitslosigkeitsphase 1997 & \multicolumn{2}{|c|}{0.22} & \multicolumn{2}{|c|}{0.18} \\
\hline Beginn der Arbeitslosigkeitsphase 1998 & \multicolumn{2}{|c|}{0.14} & \multicolumn{2}{|c|}{0.14} \\
\hline Beginn der Arbeitslosigkeitsphase 1999 & \multicolumn{2}{|c|}{0.14} & \multicolumn{2}{|c|}{0.11} \\
\hline Ausbildungsdauer in Jahren & \multicolumn{2}{|c|}{$\begin{array}{l}11.08 \\
(2.54)\end{array}$} & \multicolumn{2}{|c|}{$\begin{array}{l}12.11 \\
(1.89)\end{array}$} \\
\hline Bisherige Erwerbsdauer seit 1992 (in Monaten) & \multicolumn{2}{|c|}{$\begin{array}{c}36.88 \\
(23.79)\end{array}$} & \multicolumn{2}{|c|}{$\begin{array}{c}36.55 \\
(23.17)\end{array}$} \\
\hline Vorherige Erwerbstätigkeit (1: ja) & \multicolumn{2}{|c|}{0.68} & \multicolumn{2}{|c|}{0.72} \\
\hline $\begin{array}{l}\text { bisherige Arbeitslosigkeitsdauer seit } 1992 \text { ohne die } \\
\text { aktuelle Episode (in Monaten) }\end{array}$ & \multicolumn{2}{|c|}{$\begin{array}{c}6.71 \\
(11.05)\end{array}$} & \multicolumn{2}{|c|}{$\begin{array}{c}12.43 \\
(14.37)\end{array}$} \\
\hline Alter in Jahren im Jahr des Arbeitslosigkeitsbeginns & \multicolumn{2}{|c|}{$\begin{array}{c}37.20 \\
(11.64)\end{array}$} & \multicolumn{2}{|c|}{$\begin{array}{c}40.53 \\
(11.07)\end{array}$} \\
\hline Ausländer (1:ja) & \multicolumn{2}{|c|}{0.30} & \multicolumn{2}{|c|}{0.005} \\
\hline Frau (1: ja) & \multicolumn{2}{|c|}{0.43} & \multicolumn{2}{|c|}{0.49} \\
\hline Anzahl der Kinder zwischen 5-14 Jahren im Haushalt & $\begin{array}{c}0.36 \\
(0.66)\end{array}$ & $\begin{array}{c}0.40 \\
(0.79)\end{array}$ & $\begin{array}{c}0.64 \\
(0.83)\end{array}$ & $\begin{array}{c}0.45 \\
(0.75)\end{array}$ \\
\hline $\begin{array}{l}\text { Anzahl der Kleinkinder zwischen 0-4 Jahren im } \\
\text { Haushalt }\end{array}$ & $\begin{array}{c}0.18 \\
(0.45)\end{array}$ & $\begin{array}{c}0.20 \\
(0.49)\end{array}$ & $\begin{array}{c}0.13 \\
(0.37)\end{array}$ & $\begin{array}{c}0.10 \\
(0.34)\end{array}$ \\
\hline $\begin{array}{l}\text { Einkommen des Ehepartners (1: Ehep. vollbeschäftigt } \\
\text { oder in Rente / Vorruhestand) }\end{array}$ & 0.44 & 0.11 & 0.52 & 0.28 \\
\hline Unterstützungsanspruch (1:ja) & \multicolumn{2}{|c|}{0.79} & \multicolumn{2}{|c|}{0.89} \\
\hline soziales Engagement & \multicolumn{2}{|c|}{$\begin{array}{c}2.47 \\
(1.01)\end{array}$} & \multicolumn{2}{|c|}{$\begin{array}{c}2.47 \\
(1.00)\end{array}$} \\
\hline internale Kontrolleinstellung ( $1: \mathrm{ja})$ & & & & \\
\hline $\begin{array}{l}\text { Bedeutung des berufl. Erfolges für Zufriedenheit } \\
\text { (1: sehr wichtig) }\end{array}$ & & & & \\
\hline Mobilitätsbereitschaft aus fam./beruf. Gründen (1: ja) & & & & \\
\hline Länge der Arbeitslosigkeitsepisode (in Monaten) & & & & \\
\hline
\end{tabular}

In Klammern unter den Mittelwerten sind die Standardabweichungen der Variablen angegeben, falls es sich nicht um Anteilswerte handelt. 
Tabelle 5.1: $\quad$ Nichtproportionale Coxregressionen für Westdeutschland

\begin{tabular}{|c|c|c|c|c|c|}
\hline & & Modell 1 & Modell 2 & Modell 3 & Modell 4 \\
\hline Regionale AL-Quote & $(-)$ & $-0.0409 * *$ & $-0.0763 * * *$ & $-0.0777 * * *$ & $-0.0796 * * *$ \\
\hline Jahr des Beginns: 1999 & & -0.2622 & -0.0738 & -0.0541 & -0.0443 \\
\hline Jahr des Beginns: 1998 & & 0.0176 & 0.1668 & 0.1873 & 0.1932 \\
\hline Jahr des Beginns: 1997 & & 0.1136 & $0.2625 *$ & $0.2930 * *$ & $0.2928 * *$ \\
\hline Jahr des Beginns: 1996 & & -0.0513 & 0.0358 & 0.1248 & 0.0613 \\
\hline Jahr des Beginns: 1995 & & 0.0160 & 0.0627 & 0.0529 & 0.0491 \\
\hline Ausbildungsdauer & $(+)$ & & $0.0690 * * *$ & $0.0738 * * *$ & $0.0690 * * *$ \\
\hline Bisherige Erwerbsdauer & $(+)$ & & $0.0023 * *$ & 0.0011 & 0.0005 \\
\hline Vorherige Erwerbstätigkeit & $(+)$ & & $0.4436 * * *$ & $0.4094 * * *$ & $0.4175 * * *$ \\
\hline Bisherige AL-Dauer & $(-)$ & & -0.0009 & -0.0013 & -0.0018 \\
\hline Alter & $(-)$ & & $-0.0330 * * *$ & $-0.0333 * * *$ & $-0.0323 * * *$ \\
\hline Ausländer & $(-)$ & & $-0.4856 * * *$ & $-0.4958 * * *$ & $-0.4626 * * *$ \\
\hline Frau & $(-)$ & & $-0.2815 * * *$ & $-0.2336 * *$ & $-0.2528 * * *$ \\
\hline Kinder $(+)$ & $(-)$ & & & -0.2114 & -0.2079 \\
\hline Kleinkinder (†) & $(-)$ & & & $-1.3618 * * *$ & $-1.3490 * * *$ \\
\hline Einkommen des Ehepartners (ㅇ) & $(-)$ & & & 0.1601 & 0.1700 \\
\hline Kinder $(ð)$ & $(-)$ & & & $0.1138 *$ & $0.1093 *$ \\
\hline Kleinkinder $(ð))$ & $(-)$ & & & 0.0171 & 0.0086 \\
\hline Einkommen des Ehepartners $(ð)$ & $(-)$ & & & 0.0139 & -0.0226 \\
\hline Unterstützungsanspruch & $(-)$ & & & $-0.3132 * *$ & $-0.3147 * *$ \\
\hline Soziales Engagement & $(+)$ & & & & 0.0209 \\
\hline Internale Kontrolleinstellung & $(+)$ & & & & $0.1582 * *$ \\
\hline Bedeutung des berufl. Erfolges & $(+)$ & & & & -0.0766 \\
\hline Mobilitätsbereitschaft & $(+)$ & & & & -0.1807 \\
\hline Alter im Zeitverlauf(ZV) & & & $-0.0075 * *$ & $-0.0075 * *$ & $-0.0074 * *$ \\
\hline Kinder (†) im ZV & & & & $0.1683 *$ & $0.1787 * *$ \\
\hline Kleinkinder (P) im ZV & & & & $0.4462 * * *$ & $0.4589 * * *$ \\
\hline Unterstützungsanspruch im ZV & & & & $0.1778 *$ & $0.1738 *$ \\
\hline Mobilitätsbereitschaft im ZV & & & & & $0.1637 * *$ \\
\hline Log-Likelihood (Ausgang: - 5389. & $.09)^{1}$ & $-5382.88 *$ & $-5239.63 * * *$ & $-5222.52 * * *$ & $-5217.07 *$ \\
\hline Pseudo-R ${ }^{22}$ : & & 0.0015 & 0.0277 & 0.0311 & 0.0319 \\
\hline
\end{tabular}

Signifikanzniveaus: $* * * 1 \%, * * 5 \%, * 10 \%$ (zweiseitiger Test); $\mathrm{n}=1413$

${ }^{1}$ Das Signifikanzniveau entspricht dem „Log-Likelihood-Ratio-Test“. Der Wert der Teststatistik ergibt sich aus der doppelten Log-Likelihood-Differenz zwischen zwei Modellen, die Freiheitsgrade entsprechen der Anzahl zusätzlicher Variablen und die Teststatistik folgt näherungsweise einer Chi-Quadrat-Verteilung. Getestet wird die Nullhypothese, dass es zu keiner Verbesserung des „Model Fit“ durch die Aufnahme weiterer Kovariablen kommt.

${ }^{2}$ Der Wert des Pseudo- $\mathrm{R}^{2}$ ist nicht als Anteil der erklärten Varianz zu verstehen sondern gibt die relative Verminderung des Log Likelihood Wertes des jeweiligen Modells bezogen auf das Modell ohne Kovariablen an (Blossfeld et al. 1986). 
Tabelle 5.2: Nichtproportionale Coxregressionen für Ostdeutschland

\begin{tabular}{|c|c|c|c|c|c|}
\hline & & Modell 1 & Modell 2 & Modell 3 & Modell 4 \\
\hline Regionale AL-Quote & $(-)$ & $-0.0142 * *$ & -0.0128 & -0.0155 & -0.0227 \\
\hline Jahr des Beginns: 1999 & & $-0.6934 * * *$ & $-0.4159 * *$ & -0.3037 & -0.2810 \\
\hline Jahr des Beginns: 1998 & & $-0.2365 *$ & -0.1849 & -0.1078 & -0.0854 \\
\hline Jahr des Beginns: 1997 & & $-0.3368 * * *$ & -0.1815 & -0.1178 & -0.1013 \\
\hline Jahr des Beginns: 1996 & & $-0.2816 * * *$ & -0.1486 & -0.1146 & -0.1135 \\
\hline Jahr des Beginns: 1995 & & $-0.2601 * *$ & -0.1384 & -0.1139 & -0.1306 \\
\hline Ausbildungsdauer & $(+)$ & & $0.0487 * * *$ & $0.0481 * * *$ & $0.0427 * *$ \\
\hline Bisherige Erwerbsdauer & $(+)$ & & $0.0045^{*}$ & 0.0030 & 0.0028 \\
\hline Vorherige Erwerbstätigkeit & $(+)$ & & $0.2626 * * *$ & $0.2288 * * *$ & $0.2358 * * *$ \\
\hline Bisherige AL-Dauer & $(-)$ & & $-0.0212 * * *$ & $-0.0219 * * *$ & $-0.0220 * * *$ \\
\hline Alter & $(-)$ & & $-0.0380 * * *$ & $-0.0410 * * *$ & $-0.0415 * * *$ \\
\hline Ausländer & $(-)$ & & -0.2346 & -0.3700 & -0.4239 \\
\hline Frau & $(-)$ & & $-0.7734 * * *$ & $-0.6441 * * *$ & $-0.6420 * * *$ \\
\hline Kinder $(+)$ & $(-)$ & & & -0.0246 & -0.0166 \\
\hline Kleinkinder $(+)$ & $(-)$ & & & $-1.3995 * * *$ & $-1.3916^{* * *}$ \\
\hline Einkommen des Ehepartners ( $($ ) & $(-)$ & & & 0.0455 & 0.0401 \\
\hline Kinder $(ð)$ & $(-)$ & & & -0.1355 & -0.1463 \\
\hline Kleinkinder $(\precsim)$ & $(-)$ & & & -0.0829 & -0.0758 \\
\hline Einkommen des Ehepartners $(\precsim)$ & $(-)$ & & & $0.2577 * * *$ & $0.2469 * *$ \\
\hline Unterstützungsanspruch & $(-)$ & & & $-0.4879 * * *$ & $-0.4837 * * *$ \\
\hline Soziales Engagement & $(+)$ & & & & $0.0619 *$ \\
\hline Internale Kontrolleinstellung & $(+)$ & & & & 0.0027 \\
\hline Bedeutung des berufl. Erfolges & $(+)$ & & & & 0.0165 \\
\hline Mobilitätsbereitschaft & $(+)$ & & & & -0.0569 \\
\hline Bisherige AL-Dauer im Zeitverlauf & & & $0.0079 * * *$ & $0.0075 * * *$ & $0.0075 * * *$ \\
\hline Frau im ZV & & & $0.2179 * * *$ & $0.2202 * * *$ & $0.2196 * * *$ \\
\hline Kleinkinder (†) im ZV & & & & $0.5066^{* * *}$ & $0.5016^{* * *}$ \\
\hline Kinder $(ð) \operatorname{im~ZV~}$ & & & & $0.1256^{* *}$ & $0.1285 * *$ \\
\hline Unterstützungsanspruch im ZV & & & & $0.2235 * *$ & $0.2244 * *$ \\
\hline Log-Likelihood (Ausgang: - 6917. & 7.93) & $-6903.27 * * *$ & $-6767.37 * * *$ & $-6748.46 * * *$ & -6746.47 \\
\hline Pseudo-R ${ }^{2}$ & & 0.0021 & 0.0218 & 0.0245 & 0.0248 \\
\hline
\end{tabular}

Signifikanzniveaus: *** $1 \%, * * 5 \%, * 10 \%$ (zweiseitiger Test); $\mathrm{n}=1557$ 


\section{$7 \quad$ Literaturverzeichnis}

Andreß, Hans-Jürgen und Wolfgang Strengmann-Kuhn (1997): Warum arbeiten, wenn der Staat zahlt? Über das Arbeitsangebot unterer Einkommensschichten. Zeitschrift für Sozialreform 43: 505-526.

Barbieri, Paolo, Serge Paugam und Helen Russell (2000): Social Capital and Exits from Unemployment. 200-271 in: Duncan Gallie and Serge Paugam (Hg.): Welfare Regimes and the Experience of Unemployement in Europe. Oxford, New York: Oxford University Press.

Becker, Gary S. (1964): Human Capital. A Theoretical and Empirical Analysis, with Special Reference to Education. New York, London: Columbia University Press.

Becker, Gary S. (1996): Familie, Gesellschaft und Politik. Tübingen: Mohr.

Bender, Stefan, Ludwig Fahrmeir und Stefan Lang (2000): Determinanten der Arbeitslosigkeitsdauer in Westdeutschland. 79-94 in: Felix Büchel, Martin Diewald, Peter Kraus, Antje Mertens und Heike Soga (Hg.): Zwischen drinnen und draußen. Arbeitsmarktchancen und soziale Ausgrenzungen in Deutschland. Opladen: Leske und Budrich.

Blossfeld, Hans-Peter, Alfred Hamerle und Karl Ulrich Mayer (1986): Ereignisanalyse. Statistische Theorie und Anwendung in den Wirtschafts- und Sozialwissenschaften. Frankfurt, New York: Campus.

Blossfeld, Hans-Peter und Götz Rohwer (2002): Techniques of Event History Modelling. New Approaches to Causal Analysis. Second Edition. Mahwah, New Jersey: Lawrence Erlbaum Associates.

Coleman, James S. (1990): Foundations of Social Theory. Cambridge, Massachusetts: The Belknap Press of Harvard University Press.

Coleman, James S. (1991): Matching Processes in the Labor Market. Acta Sociologica 34: 3-12.

Esser, Hartmut (1999): Soziologie. Spezielle Grundlagen. Band 1: Situationslogik und Handeln. Frankfurt, New York: Campus.

Fischer, Lorenz und Günter Wiswede (2002): Grundlagen der Sozialpsychologie. 2.Auflage. München, Wien: Oldenbourg.

Freitag, Markus (2000): Soziales Kapital und Arbeitslosigkeit. Eine empirische Analyse zu den Schweizer Kantonen. Zeitschrift für Soziologie 29: 186-201.

Gilberg, Reiner, Doris Hess und Helmut Schröder (1999): Wiedereingliederung von Langzeitarbeitslosen. Chancen und Risiken im Erwerbsverlauf. Mitteilungen aus der Arbeitsmarkt- und Berufsforschung 32: 281-299.

Granovetter, Mark S. (1973): The Strength of Weak Ties. American Journal of Sociology 78: 13601380.

Granovetter, Mark S. (1974): Getting a Job. A Study of Contacts and Careers. Cambridge, Massachusetts, London: Harvard University Press.

Haisken-DeNew, John P. und Joachim R. Frick (Hg.) (2001): Desktop Companion to the German Socio-Economic Panel Study (GSOEP). Version 5.0. DIW Berlin.

Heckhausen, Heinz (1989): Motivation und Handeln. 2. Auflage. Berlin, Heidelberg, New York: Springer.

Heckman, James J. und George J. Borjas (1980): Does Unemployment Cause Future Unemployment? Definitions, Questions and Answers from a Continuous Time Model of Heterogeneity and State Dependence. Economica 47: 247-283.

Kaiser, Lutz C. und Thomal Siedler (2001): Die Dauer von Arbeitslosigkeit in Deutschland und Großbrittanien: Ein internationaler Vergleich (1990-1995). Mitteilungen aus der Arbeitsmarktund Berufsforschung 34: 402-418. 
Licht, Georg und Viktor Steiner (1991): Abgang aus der Arbeitslosigkeit, Individualeffekte und Hysteresis - Eine Panelanalyse für die Bundesrepublik Deutschland. 182-206 in: Helberger, Christof, Lutz Bellmann und Dieter Blaschke (Hg.): Erwerbstätigkeit und Arbeitslosigkeit. Analysen auf der Grundlage des Sozio-ökonomischen Panels. Beiträge zur Arbeitsmarkt- und Berufsforschung, Band 144. Nürnberg: Institut für Arbeitsmarkt- und Berufsforschung der Bundesanstalt für Arbeit.

Lindenberg, Siegwart (1985): An Assessment of the New Political Economy: Its Potential for the Social Sciences and for Sociology in Particular. Sociological Theory 3: 99-114.

Little, Daniel (1991): Varieties of Social Explanation. An Introduction to the Philosophy of Social Science. Boulder, San Francisco, Oxford: Westview Press.

Mincer, Jacob (1974): Schooling, experience and earnings. New York, London: Columbia University Press.

Narendranatha, Wiji und Mark B. Stewart (1993): Modelling the Probability of Leaving Unemployment: Competing Risks Models with Flexible Base-line Hazards. Journal of the Royal Statistical Society. Applied Statistics, Series C: 361-382.

Noll, Heinz-Herbert und Stefan Weick (2002): Informelle Kontakte für Zugang zu Jobs wichtiger als Arbeitsvermittlung. Prozesse der Arbeitssuche und Stellenfindung im europäischen Vergleich. Informationsdienst Soziale Indikatoren Juli 2002: 6-10.

Nolte, Helmut (1997): Kontrolleinstellungen zum Leben und zur Zukunft - Auswertung eines neuen, sozialpsychologischen Itemblocks im sozio-oekonomischen Panel. Diskussionspapier der Fakultät für Sozialwissenschaft der Ruhr-Universität Bochum.

Pötter, Ulrich und Götz Rohwer (1999): TDA User's Manual. Ruhr-Universität Bochum.

Roed, Knut und Tao Zhang (2003): Does Unemployment Compensation Affect Unemployment Duration? The Economic Journal 113: 190-206.

Schupp, Jürgen und Gert Wagner (2002): Maintenance of and innovation in long-term panel studies: The case of the German Socio-Economic Panel (SOEP). Allgemeines Statistisches Archiv 86: 163-175.

Sprengers, Maarten (1992): Explaining Unemployment Duration. An Integrative Approach. Utrecht (kein Verlag).

Thurow, Lester C. (1975): Generating Inequality. Mechanisms of Distribution in the U.S. Economy. New York: Basic Books.

Wolbers, Maarten H.J. (2000): The Effects of Level of Education on Mobility between Employment and Unemployment in the Netherlands. European Sociological Review 16: 185-200.

Yamaguchi, Kazuo (1991): Event History Analysis. Newbury Park, London, New Delhi: Sage.

Zimmermann, Klaus F. (1985): Familienökonomie. Theoretische und empirische Untersuchungen zur Frauenerwerbstätigkeit und Geburtenentwicklung. Berlin, Heidelberg, New York: Springer. 


\section{Anhang A: Operationalisierungen}

\section{Erwerbsbiographie}

Es wird jährlich retrospektiv der Erwerbsstatus jeder interviewten Person für die einzelnen Monate des Vorjahres abgefragt. Hierbei kreuzen die Befragten auf einer kalendarischen Übersicht für jeden Monat ihren jeweiligen Status an. Mögliche Zustände sind:

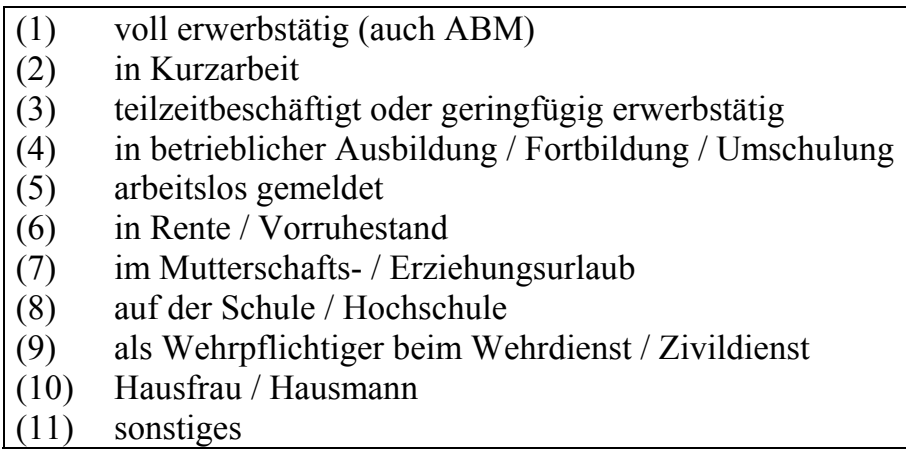

Diese Angaben liegen als Episodendaten vor, wobei der Typ, Beginn und Ende (in Monaten) der Episoden kodiert sind. Die Zählung der Monate beginnt im Januar 1983 mit dem Wert 1, der Dezember 1999 ist dementsprechend der Monat 204. In Anlehnung an Licht und Steiner (1991) werden die Zustände 1-4 zur Kategorie „erwerbstätig“ zusammengefasst, in die Kategorie „arbeitslos“ fallen die Personen, die angeben, arbeitslos gemeldet gewesen zu sein. Alle übrigen Zustände bilden die Kategorie „nicht erwerbstätig“. Aus dieser Unterteilung ergeben sich zwei mögliche Anschlusszustände an eine Phase der Arbeitslosigkeit. Der Anschlusszustand „Erwerbstätigkeit“ tritt dann ein, wenn im selben oder im darauffolgenden Monat nach Ende einer Arbeitslosigkeitsphase eine Erwerbstätigkeit aufgenommen wird, andernfalls liegt der Anschlusszustand „Nichterwerbstätigkeit“" vor.

Die „bisherige Erwerbsdauer“ beinhaltet die Anzahl der Monate, in der die arbeitslose Person seit dem Januar 1992 entweder voll erwerbstätig, in Kurzarbeit, teilzeitbeschäftigt oder geringfügig beschäftigt war. Mittels dieser Anzahl der Monate soll die bisherige Berufserfahrung einer Person gemessen werden. Nicht mitberücksichtigt werden Phasen der betrieblichen Ausbildung, Fortbildung oder Umschulung. Die dichotome Variable „vorherige Erwerbstätigkeit“ nimmt den Wert 1 an, wenn im Monat vor oder im selben Monat des Beginns einer Arbeitslosigkeitsepisode eine Phase endet, in der die Untersuchungsperson voll erwerbstätig, in Kurzarbeit, teilzeitbeschäftigt oder geringfügig beschäftigt war. Andernfalls nimmt sie den Wert 0 an. Die „bisherige Arbeitslosigkeitsdauer“ ergibt sich aus der Anzahl der Monate, die die Untersuchungsperson seit dem Januar 1992 in Arbeitslosigkeit verbracht hat, wobei die Dauer der aktuellen Periode nicht mit berücksichtig wird.

\section{Allgemeine Ausbildung}

Jedes Jahr wird den beteiligten Personen die Frage gestellt, ob Sie im vorherigen Jahr eine Schule, eine Berufsausbildung oder ein Hochschulstudium abgeschlossen haben. Zusätzlich wird jede in das Panel neu eintretende Person nach ihren bisherigen Bildungsabschlüssen befragt. Somit liegt für jedes Befragungsjahr der formal höchste Bildungsabschluss vor. Für westdeutsche Abschlüsse bedeutet dies:

\begin{tabular}{|l|l|}
\hline Schulausbildung & Jahre \\
\hline kein Abschluss & 7 \\
Hauptschule & 9 \\
Realschule & 10 \\
\hline
\end{tabular}




\begin{tabular}{|l|l|}
\hline Fachhochschulreife & 12 \\
Abitur & 13 \\
anderer Abschluss & 10 \\
zusätzliche berufliche Ausbildung & 1.5 \\
Lehre & 2 \\
Berufsfachschule & 1.5 \\
Beamtenausbildung & 3 \\
Fachhochschule & 5 \\
Universität & \\
\hline
\end{tabular}

Für eine Person, die nach dem Abitur einen Universitätsabschluss erreicht hat, ergibt sich für die „Ausbildungsdauer“ somit ein Wert von 18, unabhängig davon ob diese Person zusätzlich eine Lehre absolviert hat oder nicht (Haisken-DeNew und Frick 2001). Die Variable „Ausbildungsdauer“ enthält jeweils den letzten, vor Beginn einer Episode angegebenen Bildungsabschluss.

\section{Familiensituation}

\section{Kinderanzahl}

Dem Haushaltsvorstand wird jährlich die Frage gestellt, ob Kinder im Alter von bis zu 16 Jahren im Haushalt leben und in welchem Jahr diese Kinder geboren wurden. Die Variable „Kleinkinder“ enthält die Anzahl der Kinder zwischen 0 und 4 Jahren, die zu Beginn einer Arbeitslosigkeitsepisode in dem Haushalt leben. Die Anzahl der Kinder zwischen 5 und 14 Jahren ist in der Variable „Kinder“ enthalten.

\section{Einkommen des Ehepartners}

Lebt der Ehepartner einer Untersuchungsperson mit dieser im selben Haushalt, so wird auch diese Person in das sozioökonomische Panel aufgenommen. Die Variable „Einkommen des Ehepartners“ nimmt den Wert 1 an, wenn der im gleichen Haushalt lebende Ehepartner im Jahr des Beginns der Arbeitslosigkeit der Untersuchungsperson zwölf Monate voll erwerbstätig gewesen ist, sich zwölf Monate in Rente oder Vorruhestand befand oder die Summe der Monate in diesen beiden Zuständen zwölf ergibt. Ist dies nicht der Fall, nimmt die Variable den Wert 0 an.

\section{Anspruch auf Lohnersatzzahlungen}

Haben die Befragten in dem Jahr, in dem sie arbeitslos geworden sind, Arbeitslosengeld oder Arbeitslosenhilfe bezogen, so nimmt die Variable „Unterstützungsanspruch“ den Wert 1 an, andernfalls den Wert 0. Der Wert 1 bedeutet, dass die arbeitslose Person einen Anspruch auf Auszahlung von Lohnersatzleistungen durch die Bundesanstalt für Arbeit hat.

\section{Soziales Engagement}

In jeder Befragungswelle von 1994 bis 2000 wurde die Frage gestellt, wie oft in der freien Zeit bestimmte Tätigkeiten ausgeübt werden. In den Befragungsjahren 1994, 1996, 1997 und 1999 wurden für folgende acht Tätigkeiten die Häufigkeiten abgefragt: 
(1) Besuch von kulturellen Veranstaltungen, z.B. Konzerten, Theater, Vorträgen

(2) Kinobesuch, Besuch von Popkonzerten, Tanzveranstaltungen, Discos, Sportveranstaltungen

(3) Aktiver Sport

(4) Geselligkeit mit Freunden, Verwandten oder Nachbarn

(5) Mithelfen, wenn bei Freunden, Verwandten oder Nachbarn etwas zu tun ist

(6) Ehrenamtliche Tätigkeiten in Vereinen, Verbänden oder sozialen Diensten

(7) Beteiligung in Bürgerinitiativen, in Parteien, in der Kommunalpolitik

(8) Kirchgang, Besuch religiöser Veranstaltungen

Die korrespondierenden Fragen in den Jahren 1995 und 1998 umfassten jeweils 16 abgefragte Tätigkeiten, die zwischen diesen beiden Jahren aber voneinander abwichen. Sowohl die Häufigkeit ehrenamtlicher Tätigkeiten in Vereinen, Verbänden oder sozialen Diensten (6) als auch die Häufigkeit einer Beteiligung in Bürgerinitiativen, in Parteien und in der Kommunalpolitik (7) wurden in beiden Jahren abgefragt.

Für die Befragungswellen in den Jahren 1994, 1996, 1997 und 1999 standen den Befragten vier Antwortkategorien zu Verfügung: jede Woche (1), jeden Monat (2), seltener (3) oder nie (4): In den Befragungswellen der Jahre 1995 und 1998 waren es fünf Kategorien: täglich (1), jede Woche (2), jeden Monat (3), seltener (4) oder nie (5).

Für die Jahre 1995 und 1998 habe ich die Kategorien (1) und (2) zusammengefasst, um über alle Wellen eine einheitliche Kodierung der Häufigkeiten zu erreichen. Nach einer Umkodierung (aus 1 wird 4 etc.) wurde für jeden Befragungszeitpunkt ein additiver Index gebildet. Wurde eine der beiden Fragen nicht beantwortet, wurde dieser fehlende Wert durch die Ausprägung 1, also die Bedeutung „nie“, ersetzt. Der Wertebereich der Variablen „,soziales Engagement“ umfasst die Werte 2 bis 8. Wie die Variable „Ausbildungsdauer“ trägt auch die Variable „soziales Engagement“ den letzten gültigen Wert, den der Index vor dem Beginn einer Episode annimmt.

\section{Internale Kontrolleinstellung}

In den Befragungswellen der Jahre 1994 bis 1996 wurden die Befragten zu einer Reihe von Aussagen nach ihrer Zustimmung befragt. Sie konnten jeweils voll zustimmen (1), eher zustimmen (2), eher nicht zustimmen (3) oder überhaupt nicht zustimmen (4).

Die verschiedenen Aussagen waren:

\begin{tabular}{|l}
\hline (1) Ich kann ziemlich viel von dem, was in meinem Leben passiert, selbst bestimmen \\
(2) Planen macht einen Menschen nur unglücklich, da Pläne sich kaum jemals in die \\
Wirklichkeit umsetzen lassen \\
(3) Mein Leben wird von meinem Verhalten bestimmt \\
(4) Ich meine, keiner kann seinem Schicksal entgehen. Es kommt im Leben alles, wie es \\
kommen muss \\
(5) Wenn ich bekomme, was ich will, so geschieht dies meistens aus Glück \\
(6) Wenn ich Pläne schmiede, bin ich sicher, dass das Geplante auch Wirklichkeit wird \\
(7) Es hat wenig Sinn, fest umrissene Ziele zu verfolgen, weil doch immer etwas \\
Unerwartetes dazwischenkommt \\
(8) Es kommt doch immer anders als man denkt, man kann sich auf nichts verlassen
\end{tabular}

Ich habe die Statements der Befragten dichotomisiert und unterscheide zwischen Zustimmung und Ablehnung. Stimmt die befragte Person den Aussagen (1), (3) und (6) zu, nimmt die Variable „internale Kontrolleinstellung“ den Wert 1 an. Liegt zu mindestens einer der drei Aussagen keine Zustimmung vor, 
nimmt die Variable den Wert 0 an. Nolte et al. gehen ähnlich vor. Sie ordnen den Befragten das Einstellungsmuster „Selbstbestimmtheit“, was meiner internalen Kontrolleinstellung entspricht, allerdings bereits dann zu, wenn die Befragten zwei der drei Items zustimmen (Nolte et al. 1997). Die Variable trägt den letzten gültigen Wert, der vor dem Episodenbeginn gemessen werden kann.

\section{Mobilitätsbereitschaft}

In allen von mir berücksichtigten Befragungswellen wurde den interviewten Personen die Frage gestellt, ob sie bereit wären, aus beruflichen oder familiären Gründen den Wohnort zu wechseln. Die drei möglichen Antwortkategorien auf diese Frage sind: ja (1), kommt darauf an, will ich nicht ausschließen (2) und nein, ausgeschlossen, kaum denkbar (3).

Die Variable „Mobilitätsbereitschaft“ nimmt den Wert 1 an, wenn der Befragte auf die Frage mit ja (1) antwortet, andernfalls nimmt sie den Wert 0 an. Der letzte gültige Wert vor dem Episodenbeginn ist in der Variablen enthalten.

Im Jahre 1994 wurde in Westdeutschland kein ausländischer Panelteilnehmer meiner Stichprobe nach seiner Mobilitätsbereitschaft befragt, lediglich 7\% der von Ausländern im Jahre 1995 begonnenen Episoden enthalten gültige Werte. Diese fehlenden Werte wurden durch den Mittelwert der Mobilitätsbereitschaft der Ausländer (0.373) ersetzt.

\section{Bedeutung des beruflichen Erfolges}

In vier Befragungswellen zwischen 1994 und 1999 wurde die Frage gestellt, wie wichtig bestimmte Bereiche des Lebens für die Zufriedenheit des Befragten sind. Einer dieser abgefragten Bereiche war der berufliche Erfolg. 1994, 1998 und 1999 wurde die Frage wie folgt formuliert:

„Welche der nachfolgenden Bereiche sind für Ihr Wohlbefinden und Ihre Zufriedenheit sehr wichtig, wichtig, weniger wichtig oder ganz unwichtig? Wie wichtig ist für Ihr Wohlbefinden und Ihre Zufriedenheit“ ... ? Diese Wichtigkeit wurde 1998 und 1999 für folgende Bereiche abgefragt:

\begin{tabular}{|ll|}
\hline$(1)$ & die Arbeit \\
$(2)$ & die Familie \\
$(3)$ & der Freundeskreis \\
$(4)$ & das Einkommen \\
$(5)$ & die Wohnung \\
$(6)$ & der Einfluß auf politische Entscheidungen \\
$(7)$ & der Erfolg im Beruf \\
$(8)$ & die Freizeit \\
$(9)$ & die Gesundheit \\
$(10)$ & der Schutz der natürlichen Umwelt \\
$(11)$ & der Glaube, die Religion \\
$(12)$ & die Wohngegend / die Wohnlage \\
$(13)$ & die Mobilität, also daß man überall schnell hinkommt \\
\hline
\end{tabular}

1994 umfasste die Frage die Bereiche (1) bis (11).

Im Jahre 1995 weicht die Formulierung der Frage von der obigen leicht ab: „Verschiedenen Menschen sind verschiedene Dinge im Leben wichtig. Sind für Sie persönlich die folgenden Dinge heute sehr wichtig, wichtig, weniger wichtig oder ganz unwichtig?“ 
(1) Sich etwas leisten können

(2) Für andere da sein

(3) Sich selbst verwirklichen

(4) Erfolg im Beruf haben

(5) Ein eigenes Haus haben

(6) Eine glückliche Ehe / Partnerschaft führen

(7) Kinder haben

(8) Viel mit Freunden zusammen sein

(9) Sich politisch, gesellschaftlich einsetzen

(10) Die Welt sehen, viele Reisen machen

Es stehen jeweils vier Antwortkategorien zur Verfügung: sehr wichtig (1), wichtig (2), weniger wichtig (3) und ganz unwichtig (4). Die Variable „Bedeutung des beruflichen Erfolges“ nimmt den Wert 1 an, wenn die Befragten angeben, der berufliche Erfolg sei für ihre Zufriedenheit sehr wichtig, andernfalls nimmt die Variable den Wert 0 an. Auch die Variable „Bedeutung des beruflichen Erfolges“ trägt den letzten gültigen Wert, der vor dem Beginn der jeweiligen Episode aufgenommen wurde.

\section{Regionale Arbeitslosenquote}

Für die befragten Personen liegt die Bundeslandzugehörigkeit zu jedem Befragungszeitpunkt vor. Hierbei sind das Saarland und Rheinland-Pfalz zu einer Region zusammengefasst. Es wird zwischen Berlin Ost und Berlin West unterschieden, ich fasse beide Gebiete zum Bundesland Berlin zusammen. Im Statistischen Jahrbuch 2001 sind die jährlichen, durchschnittlichen Arbeitslosigkeitsquoten der Jahre 1991 bis 2000 für jedes Bundesland dokumentiert. Diese Quote gibt jeweils die Arbeitslosen in Prozent der abhängigen zivilen Erwerbspersonen an (Statistisches Jahrbuch 2001). Die länderspezifische Arbeitslosenquote wird entsprechend dem Jahr, in dem eine Arbeitslosigkeitsepisode beginnt, in der Variablen ,regionale Arbeitslosenquote“ aufgenommen. 


\section{Anhang B: Interaktionen mit der Zeit}

Tabelle B.1: Geschätzte monatliche Effekte der Kovariablen Alter und Anzahle der Kinder im Haushalt weiblicher Arbeitsloser in Westdeutschland

\begin{tabular}{|c|c|c|c|c|c|c|c|}
\hline \multirow[t]{2}{*}{$\underline{\text { Dauer }}$} & \multirow[t]{2}{*}{$\ln ($ Dauer $)$} & \multicolumn{3}{|c|}{$\underline{\text { Alter in Jahren }}$} & \multicolumn{3}{|c|}{$\underline{\text { Anzahl der Kinder (Frauen) }}$} \\
\hline & & 18 & 30 & 50 & 1 & 2 & 3 \\
\hline 1 & 0,00 & 0,00 & $-0,39$ & $-1,03$ & $-0,21$ & $-0,42$ & $-0,62$ \\
\hline 2 & 0,69 & 0,00 & $-0,45$ & $-1,20$ & $-0,08$ & $-0,17$ & $-0,25$ \\
\hline 3 & 1,10 & 0,00 & $-0,49$ & $-1,29$ & $-0,01$ & $-0,02$ & $-0,03$ \\
\hline 4 & 1,39 & 0,00 & $-0,51$ & $-1,36$ & 0,04 & 0,08 & 0,12 \\
\hline 6 & 1,79 & 0,00 & $-0,55$ & $-1,46$ & 0,11 & 0,22 & 0,34 \\
\hline 8 & 2,08 & 0,00 & $-0,57$ & $-1,53$ & 0,16 & 0,33 & 0,49 \\
\hline 10 & 2,30 & 0,00 & $-0,59$ & $-1,58$ & 0,20 & 0,41 & 0,61 \\
\hline 12 & 2,48 & 0,00 & $-0,61$ & $-1,62$ & 0,24 & 0,47 & 0,71 \\
\hline 14 & 2,64 & 0,00 & $-0,62$ & $-1,66$ & 0,26 & 0,53 & 0,79 \\
\hline 16 & 2,77 & 0,00 & $-0,63$ & $-1,69$ & 0,29 & 0,58 & 0,86 \\
\hline 18 & 2,89 & 0,00 & $-0,64$ & $-1,72$ & 0,31 & 0,62 & 0,93 \\
\hline 20 & 3,00 & 0,00 & $-0,65$ & $-1,74$ & 0,33 & 0,65 & 0,98 \\
\hline 22 & 3,09 & 0,00 & $-0,66$ & $-1,77$ & 0,34 & 0,69 & 1,03 \\
\hline 24 & 3,18 & 0,00 & $-0,67$ & $-1,79$ & 0,36 & 0,72 & 1,08 \\
\hline 26 & 3,26 & 0,00 & $-0,68$ & $-1,81$ & 0,37 & 0,75 & 1,12 \\
\hline 28 & 3,33 & 0,00 & $-0,68$ & $-1,82$ & 0,39 & 0,78 & 1,16 \\
\hline 30 & 3,40 & 0,00 & $-0,69$ & $-1,84$ & 0,40 & 0,80 & 1,20 \\
\hline
\end{tabular}

Tabelle B.2: Geschätzte monatliche Effekte der Anzahl der Kleinkinder im Haushalt weiblicher Arbeitsloser, des Unterstützungsanspruch und der Mobilitätsbereitschaft in Westdeutschland

\begin{tabular}{|c|c|c|c|c|c|c|}
\hline \multirow[t]{2}{*}{$\underline{\text { Dauer }}$} & \multirow[t]{2}{*}{$\ln$ (Dauer) } & \multicolumn{3}{|c|}{$\frac{\text { Anzahl derKleinkinder }}{\underline{(\text { Frauen })}}$} & \multirow{2}{*}{$\frac{\text { Unterstützungs- }}{\frac{\text { anspruch }}{\mathrm{ja}}}$} & \multirow{2}{*}{$\frac{\frac{\text { Mobilitäts- }}{\text { bereitschaf }}}{\text { ja }}$} \\
\hline & & 1 & 2 & 3 & & \\
\hline 1 & 0,00 & $-1,35$ & $-2,70$ & $-4,05$ & $-0,31$ & $-0,18$ \\
\hline 2 & 0,69 & $-1,03$ & $-2,06$ & $-3,09$ & $-0,19$ & $-0,07$ \\
\hline 3 & 1,10 & $-0,84$ & $-1,69$ & $-2,53$ & $-0,12$ & 0,00 \\
\hline 4 & 1,39 & $-0,71$ & $-1,43$ & $-2,14$ & $-0,07$ & 0,05 \\
\hline 6 & 1,79 & $-0,53$ & $-1,05$ & $-1,58$ & 0,00 & 0,11 \\
\hline 8 & 2,08 & $-0,39$ & $-0,79$ & $-1,18$ & 0,05 & 0,16 \\
\hline 10 & 2,30 & $-0,29$ & $-0,58$ & $-0,88$ & 0,09 & 0,20 \\
\hline 12 & 2,48 & $-0,21$ & $-0,42$ & $-0,63$ & 0,12 & 0,23 \\
\hline 14 & 2,64 & $-0,14$ & $-0,28$ & $-0,41$ & 0,14 & 0,25 \\
\hline 16 & 2,77 & $-0,08$ & $-0,15$ & $-0,23$ & 0,17 & 0,27 \\
\hline 18 & 2,89 & $-0,02$ & $-0,05$ & $-0,07$ & 0,19 & 0,29 \\
\hline 20 & 3,00 & 0,03 & 0,05 & 0,08 & 0,21 & 0,31 \\
\hline 22 & 3,09 & 0,07 & 0,14 & 0,21 & 0,22 & 0,33 \\
\hline 24 & 3,18 & 0,11 & 0,22 & 0,33 & 0,24 & 0,34 \\
\hline 26 & 3,26 & 0,15 & 0,29 & 0,44 & 0,25 & 0,35 \\
\hline 28 & 3,33 & 0,18 & 0,36 & 0,54 & 0,26 & 0,36 \\
\hline 30 & 3,40 & 0,21 & 0,42 & 0,64 & 0,28 & 0,38 \\
\hline
\end{tabular}


Tabelle B.3: Geschätzte monatliche Effekte der bisherigen Arbeitslosigkeitsdauer und Anzahl der Kleinkinder im Haushalt weiblicher Arbeitsloser in Ostdeutschland

\begin{tabular}{|c|c|c|c|c|c|c|c|}
\hline \multirow[t]{3}{*}{ Dauer } & \multirow[t]{3}{*}{$\ln ($ Dauer $)$} & \multicolumn{3}{|c|}{ vorherige Arbeitslosigkeitsdauer in } & \multicolumn{3}{|c|}{ Anzahl der Kleinkinder (Frauen) } \\
\hline & & \multicolumn{3}{|c|}{ Monaten } & & & \\
\hline & & 5 & 12 & 24 & 1 & 2 & 3 \\
\hline 1 & 0,00 & $-0,11$ & $-0,26$ & $-0,53$ & $-1,39$ & $-2,78$ & $-4,17$ \\
\hline 2 & 0,69 & $-0,08$ & $-0,20$ & $-0,40$ & $-1,04$ & $-2,09$ & $-3,13$ \\
\hline 3 & 1,10 & $-0,07$ & $-0,17$ & $-0,33$ & $-0,84$ & $-1,68$ & $-2,52$ \\
\hline 4 & 1,39 & $-0,06$ & $-0,14$ & $-0,28$ & $-0,70$ & $-1,39$ & $-2,09$ \\
\hline 6 & 1,79 & $-0,04$ & $-0,10$ & $-0,21$ & $-0,49$ & $-0,99$ & $-1,48$ \\
\hline 8 & 2,08 & $-0,03$ & $-0,08$ & $-0,15$ & $-0,35$ & $-0,70$ & $-1,05$ \\
\hline 10 & 2,30 & $-0,02$ & $-0,06$ & $-0,11$ & $-0,24$ & $-0,47$ & $-0,71$ \\
\hline 12 & 2,48 & $-0,02$ & $-0,04$ & $-0,08$ & $-0,15$ & $-0,29$ & $-0,44$ \\
\hline 14 & 2,64 & $-0,01$ & $-0,03$ & $-0,05$ & $-0,07$ & $-0,14$ & $-0,20$ \\
\hline 16 & 2,77 & $-0,01$ & $-0,01$ & $-0,03$ & 0,00 & 0,00 & 0,00 \\
\hline 18 & 2,89 & 0,00 & 0,00 & $-0,01$ & 0,06 & 0,12 & 0,17 \\
\hline 20 & 3,00 & 0,00 & 0,01 & 0,01 & 0,11 & 0,22 & 0,33 \\
\hline 22 & 3,09 & 0,01 & 0,01 & 0,03 & 0,16 & 0,32 & 0,48 \\
\hline 24 & 3,18 & 0,01 & 0,02 & 0,04 & 0,20 & 0,41 & 0,61 \\
\hline 26 & 3,26 & 0,01 & 0,03 & 0,06 & 0,24 & 0,49 & 0,73 \\
\hline 28 & 3,33 & 0,01 & 0,04 & 0,07 & 0,28 & 0,56 & 0,84 \\
\hline 30 & 3,40 & 0,02 & 0,04 & 0,08 & 0,31 & 0,63 & 0,94 \\
\hline
\end{tabular}

Tabelle B.4: Geschätzte monatliche Effekte des Geschlechts, der Anzahl der Kinder im Haushalt männlicher Arbeitsloser und des Unterstützungsanspruch in Ostdeutschland

\begin{tabular}{|c|c|c|c|c|c|c|}
\hline \multirow[t]{2}{*}{$\underline{\text { Dauer }}$} & \multirow[t]{2}{*}{$\ln ($ Dauer $)$} & \multirow{2}{*}{$\frac{\text { Frau }}{\text { ja }}$} & \multicolumn{3}{|c|}{ Anzahl der Kinder (Männer) } & \multirow{2}{*}{$\frac{\text { Unterstützungs- }}{\frac{\text { anspruch }}{\text { ja }}}$} \\
\hline & & & 1 & 2 & 3 & \\
\hline 1 & 0,00 & $-0,64$ & $-0,15$ & $-0,29$ & $-0,44$ & $-0,48$ \\
\hline 2 & 0,69 & $-0,49$ & $-0,06$ & $-0,11$ & $-0,17$ & $-0,33$ \\
\hline 3 & 1,10 & $-0,40$ & $-0,01$ & $-0,01$ & $-0,02$ & $-0,24$ \\
\hline 4 & 1,39 & $-0,34$ & 0,03 & 0,06 & 0,10 & $-0,17$ \\
\hline 6 & 1,79 & $-0,25$ & 0,08 & 0,17 & 0,25 & $-0,08$ \\
\hline 8 & 2,08 & $-0,19$ & 0,12 & 0,24 & 0,36 & $-0,02$ \\
\hline 10 & 2,30 & $-0,14$ & 0,15 & 0,30 & 0,45 & 0,03 \\
\hline 12 & 2,48 & $-0,10$ & 0,17 & 0,35 & 0,52 & 0,07 \\
\hline 14 & 2,64 & $-0,06$ & 0,19 & 0,39 & 0,58 & 0,11 \\
\hline 16 & 2,77 & $-0,03$ & 0,21 & 0,42 & 0,63 & 0,14 \\
\hline 18 & 2,89 & $-0,01$ & 0,23 & 0,45 & 0,68 & 0,16 \\
\hline 20 & 3,00 & 0,02 & 0,24 & 0,48 & 0,72 & 0,19 \\
\hline 22 & 3,09 & 0,04 & 0,25 & 0,50 & 0,75 & 0,21 \\
\hline 24 & 3,18 & 0,06 & 0,26 & 0,52 & 0,79 & 0,23 \\
\hline 26 & 3,26 & 0,07 & 0,27 & 0,54 & 0,82 & 0,25 \\
\hline 28 & 3,33 & 0,09 & 0,28 & 0,56 & 0,85 & 0,26 \\
\hline 30 & 3,40 & 0,10 & 0,29 & 0,58 & 0,87 & 0,28 \\
\hline
\end{tabular}

